\title{
Exosomal microRNA-486-5p From Adipose Derived Stem Cells Alleviate Chondrocyte Apoptosis and Osteoarthritis by Attenuating Endoplasmic Reticulum Stress
}

\section{Yiming Wang}

Tongji University School of Medicine

\section{Aoyuan Fan}

Tongji University School of Medicine

Liangyu Lu

Tongji University School of Medicine

Zhangyi Pan

Tongji University School of Medicine

Min Ma

Tongji University School of Medicine

Shulin Luo

Tongji University School of Medicine

Zheng Liu

Tongji University School of Medicine

\section{Liqing Yang}

Tongji University School of Medicine

\section{Junfeng Cai}

Tongji University School of Medicine

Feng Yin ( 001 yinfeng@sina.com )

Tongji University School of Medicine https://orcid.org/0000-0002-2070-0085

\section{Research}

Keywords: Exosomes, adipose derived stem cells, microRNA-486-5p, osteoarthritis, endoplasmic reticulum stress

Posted Date: September 21st, 2021

DOI: https://doi.org/10.21203/rs.3.rs-910465/v1 
License: (c) (i) This work is licensed under a Creative Commons Attribution 4.0 International License. Read Full License 
1 Exosomal microRNA-486-5p from adipose derived stem cells alleviate chondrocyte apoptosis and osteoarthritis by attenuating endoplasmic reticulum stress

5 Yiming Wang ${ }^{1 *}$, Aoyuan Fan ${ }^{1 *}$, Liangyu Lu ${ }^{1}$, Zhangyi Pan ${ }^{1}$, Min Ma ${ }^{1}$, Shulin Luo ,

6 Zheng Liư ${ }^{1}$, Liqing Yang ${ }^{1}$, Junfeng Cai ${ }^{1 \#}$, Feng Yin ${ }^{1 \#}$

$8{ }^{1}$ Department of joint surgery, Shanghai East Hospital, Tongji University School of 9 Medicine, Shanghai, China.

11 * These authors contributed equally to this article.

\#Corresponding author: Feng Yin, Department of joint surgery, Shanghai East Hospital, Tongji University School of Medicine, Shanghai, China. E-mail: 001yinfeng@sina.com

14 Co-corresponding author: Junfeng Cai, Department of joint surgery, Shanghai East 15 Hospital, Tongji University School of Medicine, Shanghai, China. E-mail: dr_cjf@163.com

\section{Abstract}

Background: As one of the most common disabling diseases in the musculoskeletal system, osteoarthritis $(\mathrm{OA})$ is characterized with cartilage matrix degeneration and chondrocyte apoptosis. Endoplasmic reticulum (ER) stress is well known for participate in chondrocyte apoptosis and cartilage degeneration in OA progression. microRNAs 
(miRNAs) could function in cartilage homeostasis, yet limited is known regarding whether miRNA could modulate ER stress in chondrocytes. Here, we reported that exosomal microRNA-486-5p from adipose derived stem cells (ADSCs) could alleviate chondrocyte apoptosis and osteoarthritis by attenuating ER stress

Methods: ER stress markers and inflammatory cytokines were analyzed in OA knee joint by immunohistochemistry and immunofluorescence staining. IL-1 $\beta$ induced apoptosis of chondrocytes was analyzed using flow cytometry. ER stress markers and inflammatory cytokines of IL-1 induced chondrocytes were analyzed by immunofluorescence staining, western blot and ELISA. miR-486-5p mimic overexpressed ADSCs and their exosomes were validated and used to treat IL-1 $\beta$ induced chondrocytes together with miR-486-5p mimic. Different administrative methods of miR-486-5p mimic were tracked both in vitro and in vivo, and further used to treat OA model mice. OA progression of mice was analyzed by H\&E, safranin O/fast green, immunofluorescence and immunohistochemistry staining.

Results: We validated the increased inflammation and ER stress in OA synovium and cartilage, and the IL-1 $\beta$ induced chondrocyte apoptosis was through the ER stress activation. Administration of exogenous miR-486-5p could not only inhibit the ER stress, but also alleviate chondrocytes apoptosis and promote matrix regeneration. In comparison with direct administration of miR-486-5p and miR-486-5p overexpressing ADSCs, exosomes seem to be a better delivery vehicle for miRNA in modulating chondrocyte homeostasis. Our immunofluorescence and IVIS imaging data further validated the better delivery ability of exosomes through tracking the uptake of miR- 
486-5p in chondrocytes and the diffusion of miR-486-5p in the knee joint with different transportation methods. Exosomal microRNA-486-5p also showed a better effect on alleviating mice $\mathrm{OA}$.

Conclusion: Our data demonstrated that exosomes are better delivery vehicle for miR-486-5p on alleviating chondrocyte apoptosis and osteoarthritis. This study provides evidence to this efficient strategy of exosomal miRNA delivery and to the miRNA-based therapy for OA.

Keyword: Exosomes, adipose derived stem cells, microRNA-486-5p, osteoarthritis, endoplasmic reticulum stress

\section{Background}

Osteoarthritis (OA) is characterized by articular cartilage degradation, which is largely induced by the inflammatory microenvironment of the entire joint (1). Inflammatory cytokines in the OA joint can activate prolonged endoplasmic reticulum (ER) stress in chondrocytes, the major resident cell of articular cartilage, causing apoptosis (2). Inhibiting the ER stress-induced apoptosis of chondrocytes holds the potential to become a novel OA therapy.

MicroRNAs (miRNAs) are clusters of small noncoding RNAs that suppress gene expression through binding to the 3'-untranslated regions (3'-UTRs) region of the targeted mRNAs. miRNAs have emerged as a double-edged sword during the interaction with ER stress as some of them can either suppress (3) or promote (4) ER 
stress. However, whether and which miRNAs could regulate ER stress in chondrocytes remain poorly studied. Li et al. (5) reported that miR-375 could suppress autophagy and promote ER stress in chondrocytes, and the inhibition of miR-375 could attenuate OA symptoms. Yet to our knowledge, no other study has been published to clarify other miRNAs that could regulate ER stress in chondrocytes. miR-486-5p has been previously investigated in oncology and it is an important biomarker in cancer diagnosis and prognosis $(6,7)$. Recent studies have shown that the expression level of miR-486$5 p$ is related to many musculoskeletal diseases including intervertebral disc degeneration, knee OA and rheumatoid arthritis (8-10). Moreover, miR-486-5p was identified to participate in the regulation of apoptosis in nucleus pulposus cells (11). However, delivery of miR-486-5p in previous studies was through the over-expression of miR-486-5p in autologous cells by direct genetic manipulation, posing a potential risk of biological safety. Direct administration is another potential delivery method of miRNAs, but poor serum stability and low cellular uptake of the direct delivered miRNAs have remarkably restrained their clinical application (12). More effective and safer administration method is in urgent need to meet clinical requirements.

Mesenchymal stem cells (MSCs) are innovative therapeutics of OA originally based on their chondrogenic differentiation ability. Recently, it has been demonstrated that the paracrine factors including exosomes (exos), also contributes to the MSC-based OA therapy (13). Exos are subpopulation of nanoscale extracellular vesicles involved in intercellular communication, in which contain various molecules including proteins, lipids, and a variety of RNAs (miRNAs, messenger RNAs, transfer RNAs, etc.) (14). 
MSCs-derived exos have been demonstrated to benefit cartilage regeneration via increasing proliferation, diminishing apoptosis and regulating inflammatory activity (15, 16). Despite its original contents, exos are naturally capable of encapsulating and delivering cargo to modify cellular function, which emphasizes the potential usage as therapeutic delivery vehicles for miRNA-based therapy. Recent studies have indeed suggested that exos derived from both miR-92-3p and miR-140-5p-overexpressed MSCs are superior over the original MSC-derived exos for OA suppression $(17,18)$. However, it is still unclear whether exos are a better mode of conveyance when compared to the direct administration of miRNAs and MSCs.

Herein, we transduced miR-486-5p mimic into adipose derived stem cells (ADSCs) to acquire miR-486-5p mimic overexpressed ADSCs (miR-486-5p mimic ADSCs) and their exos (miR-486-5p mimic exos). We conduct a comprehensive comparison among miR-486-5p, miR-486-5p mimic ADSCs and miR-486-5p mimic exos regarding their role in chondrocyte apoptosis inhibition in vitro and OA alleviation in vivo. We believe this will provide new insight into the miRNA-cell interaction and the delivery methods of miRNAs on inhibiting chondrocytes apoptosis and OA progression.

\section{Materials and Methods}

This study was carried out in compliance with the Declaration of Helsinki.

\section{Sample collection and cell preparation}

Human subjects research was performed according to the Institutional Review Boards 
111 at Shanghai East Hospital via approved protocols with appropriate informed consent.

112 Normal synovium samples were harvested from three patients underwent exploratory

113 arthroscopy who showed no radiographic changes of the knee joint and no obvious

114 cartilage lesion during arthroscopy ( 1 male and 2 female, 68 years old on average).

115 OA synovium and articular cartilage samples were harvested from three patient

116 underwent total knee arthroplasty (1 male and 2 female, 65 years old on average).

117 Wore weight bearing area articular cartilage was used as OA cartilage, and samples

118 collected from none-weight bearing area and showed no obvious abrasion under

119 stereoscope were considered as normal articular cartilage. Samples were fixed in

120 formaldehyde, dehydrated before embedding in paraffin. Cartilage samples were

121 additionally decalcified for three days before dehydration. Samples were cut into $5 \mu \mathrm{m}$

122 thick sections.

123 Subcutaneous adipose tissues were harvested from 3 volunteers (2 male and 1

124 female, 25 years old on average). The adipose tissues were minced and sequentially

125 digested with $0.1 \%$ collagenase II (Sigma-Aldrich, St. Louis, MO, USA) for $2 \mathrm{~h}$ and $0.1 \%$

$126 \operatorname{trypsin}\left(\mathrm{Gibco}\right.$, Carlsbad, CA, USA) for $0.5 \mathrm{~h}$ at $37{ }^{\circ} \mathrm{C}$ to separate cells. After filtration

127 and centrifugation, ADSCs were seeded in growth medium [Minimum Essential

128 Medium-Alpha Modification ( $\alpha-M E M)$ containing 10\% fetal bovine serum (FBS), 100

$129 \mathrm{U} / \mathrm{ml}$ penicillin, $100 \mu \mathrm{g} / \mathrm{ml}$ streptomycin (Invitrogen, Carlsbad, CA)].

130 Human articular chondrocytes cell line was purchased from Sciencell (Catalog 131 \#4650).

132 Immunohistochemical and immunofluorescent staining 
133 For immunohistochemical staining, sections were deparaffinated, rehydrated and 134 blocked before antibody staining. Sections were then incubated with primary 135 antibodies against IL-1 $\beta$ (Affinity, AF5103), TNF- $\alpha$ (Affinity, AF7014), CHOP 136 (Proteintech, 15204-1-AP), GRP78 (Proteintech, 11587-1-AP), iNOS (Proteintech, 137 18985-1-AP) and CD163 (Proteintech, 16646-1-AP) followed by the horseradish 138 peroxidase (HRP)-conjugated Goat anti Rabbit secondary antibody (Aspen, AS-1107).

139 For immunofluorescent staining, sections were rehydrated and blocked before 140 antibody staining. Sections or cells were then incubated with primary antibodies 141 against iNOS (Proteintech, 18985-1-AP), CD163 (Proteintech, 16646-1-AP) or CHOP 142 (Proteintech, 15204-1-AP), then followed by the Cy3-labeled Goat anti Rabbit 143 secondary antibody (Aspen, AS-1109). Both sections and cells were stained with 4-6144 diamidino-2-phenylindole (DAPI, Beyotime, China) for 5 min.

145 Images were acquired using Olympus IX51 Inverted Fluorescence Microscope 146 (Olympus, Japan). qPCR, western blot and ELISA

149 For qPCR, total RNA was extracted using TRIzol reagent (Invitrogen) and was reverse150 transcribed using PrimeScript RT Reagent Kit (Takara, Japan). qPCR reaction was 151 conducted in a final volume of 20 ul containing 10 ul of HieffTM qPCR SYBR Green 152 Master Mix (YEASEN) and 2 ul of cDNA. qPCR amplification was performed using a 1537500 real-time PCR System (APPLIED Biosystems) according to the manufacturer's 154 instructions. miR-486-5p level was analyzed and normalized by U6. mRNA level of 
COL2A1, ACAN and MMP13 were assessed and GAPDH was used as the internal

control. The PCR primers used include hsa-miR-486-5p (MIMAT0002177): 5'- TGT ACT GAG CTG CCC CGA G-3' and 5'- CTC AAC TGG TGT CGT GGA GTC-3'; U6 (NR_004394.1): 5'- CTC GCT TCG GCA GCA CAT-3' and 5'- AAC GCT TCA CGAATT TGC GT-3'; GAPDH (NM_001256799.2): 5'- CAT CAT CCC TGC CTC TAC TGG-3' and 5'- GTG GGT GTC GCT GTT GAA GTC-3'; ACAN (NM_013227.3): 5'- AAG GGC GAG TGG AAT GAT GT-3' and 5'- CGC TTC TGT AGT CTG CGT TTG T-3'; COL2A1 (NM_001844.4): 5'- ATG CCA CAC TCA AGT CCC TCA-3' and 5'- GTC TCG CCA GTC TCC ATG TTG-3'; MMP13 (NM_002427.4): 5'- ATC ATG ATC TCT TTT GGAATT AAG G-3' and 5'- AAC AAG TTG TAG CCT TTG GAA CTA C-3'.

For western blot analysis, total protein was extracted from cell lysis using protein extraction kit (Byotime) and $20 \mu \mathrm{g}$ of total protein from each sample were separated

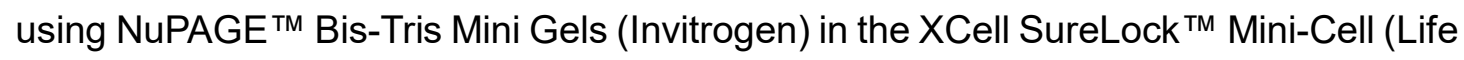
Technologies, Carlsbad, CA). Bands were transferred onto a nitrocellulose membrane using an XCell $\mathrm{II}^{\mathrm{TM}}$ Blot module (Life Technologies) at $70 \mathrm{~V}$ at $4^{\circ} \mathrm{C}$ overnight. After blocking, the membrane was incubated with primary monoclonal antibodies targeting p-PERK (bioss, bs-330R), PERK (Cell signaling technology, \#3192), p-IRE1a (abcam, ab48187), IRE1a (Cell signaling technology, \#3294), SOX9 (santa, sc-166505), GRP78 (Cell signaling technology, \#3183), type II collagen (Proteintech, 28459-1-AP), MMP13 (abcam, ab39012), cleaved Caspase-3 (abcam, ab49822) and GAPDH (abcam, ab37168) in 5\% BSA in TBST buffer at $4^{\circ} \mathrm{C}$ overnight, followed by the secondary antibody of HRP-conjugated goat anti-rabbit (Aspen, AS-1107) for $1 \mathrm{~h}$. 
$\mathrm{ECL}^{\mathrm{TM}}$ Prime Western Blotting Detection Reagents (Amersham Biosciences, Waltham, MA) were used for exposure.

The secretion of inflammatory cytokines including IL-1 $\beta$ and TNF- $\alpha$ by chondrocytes were evaluated using human ELISA kit (ELK Biotechnology, ELK1156 and ELK1190). Cell free supernatant was collected after centrifugation at $2000 \mathrm{~g}$ for $10 \mathrm{~min}$. ELISA analysis was conducted according to the manufacturer's instruction.

\section{Apoptosis and proliferation analysis}

Apoptosis was measured by flow cytometry using a FITC-conjugated Annexin $\mathrm{V}$ and propidium iodide $(\mathrm{PI})$ apoptosis kit (Invitrogen) based on the manufacturer's instructions. Briefly, cells were detached and incubated with FITC-conjugated Annexin V to stain apoptotic cells and PI to stain necrotic cells. Fluorescence was measured by a FACS Calibur (BD Biosciences) using the FCS Express software package (De Novo Software, Los Angeles, CA).

Proliferation rate was measured using Click-iT 5-ethynyl-2'-deoxyuridine (EdU) cell Proliferation Assay kit (Invitrogen). Briefly, when cells reached $50 \%$ confluence, EdU was added to the culture medium at a final concentration of $10 \mu \mathrm{M}$ and the cells were incubated at $37^{\circ} \mathrm{C}$ for $18 \mathrm{~h}$ before being fixed with $4 \%$ paraformaldehyde. Collected cells were incubated with Click-iT reaction cocktail at room temperature for $30 \mathrm{~min}$. Fluorescence was analyzed by a FACS Calibur (BD Biosciences, San Jose, CA) using the FCS Express software package (De Novo Software, Los Angeles, CA). 
miR-486-5p transduction

Passage 1 ADSCs or chondrocytes were transduced with miR-486-5p mimic, miR-486-

$5 p$ negative control mimic (NC mimic), miR-486-5p inhibitor, and miR-486-5p negative control inhibitor (NC inhibitor) using Lipofectamine ${ }^{\mathrm{TM}} 2000$ according to the manufacturer's instructions. After $24 \mathrm{~h}$ of transduction, the cells were used in the following experiments. The sequences were listed below. hsa-miR-486-5p mimics: 5'UCC UGU ACU GAG CUG CCC CGA G -3'; NC mimics: 5'- UCA CAA CCU CCU AGA AAG AGU AGA-3'; hsa-miR-486-5p inhibitor: 5'- CUC GGG GCA GCU CAG UAC AGG A -3'; NC inhibitor: 5'- UCU ACU CUU UCU AGG AGG UUG UGA -3'.

\section{Exosome isolation and characterization}

For exosome isolation, the culture medium of ADSCs was harvested and centrifuged at $2000 \mathrm{~g}$ for $30 \mathrm{~min}$ first to remove dead cells and debris. The supernatant was filtered through $0.22 \mu \mathrm{m}$ filter and transferred into a new tube. Total exosome isolation reagent (Invitrogen) was added before centrifuged at $10000 \mathrm{~g}$ for $10 \mathrm{~min}$ to obtain the exosome samples. Transmission Electron Microscopy was used to observe the exosome morphology and nanoparticle trafficking analysis was used to analyze the size of exos.

216 Exosome markers including CD9 (abcam, ab92726), CD63 (abcam, ab216130), 217 HSP70 (abcam, ab181606) were evaluated using western blot analysis. For the uptake study, exos were incubated with PKH26 (Sigma-Aldrich) for 5 min and resuspended in basal medium before incubated with chondrocytes for $12 \mathrm{~h}$. After that, chondrocytes were fixed and incubated with DAPI for 5 min. Olympus IX51 Inverted Fluorescence 
Microscope (Olympus) was used for image capture.

\section{In vitro and in vivo tracking of miR-486-5p}

miR-486-5p was tracked in both non-inflammatory and inflammatory [IL-1 $\beta(10 \mathrm{ng} / \mathrm{mL})$ in vitro and destabilization of the medial meniscus (DMM) model in vivo] environment. miR-486-5p mimic was labelled with fluorescent dye Cy3 (miR-486-5p-Cy3) and both miR-486-5p-Cy3 transduced ADSCs (miR-486-5p-Cy3 ADSCs) and exos from miR486-5p-Cy3 ADSCs (miR-486-5p-Cy3 exos) were obtained. In vitro, both normal and IL-1 $\beta$ pre-conditioned chondrocytes were treated with miR-486-5p-Cy3, miR-486-5pCy3 ADSCs (in the upper chamber of Transwell culture system) and miR-486-5p-Cy3 exos. After 6 h, 24 h and 7 days, the uptake rate of miR-486-5p-Cy3 was measured using Olympus IX51 Inverted Fluorescence Microscope (Olympus). In vivo, both normal and DMM knee joint were injected with miR-486-5p-Cy3, miR-486-5p-Сy3 ADSCs and miR-486-5p-Cy3 exos. The fluorescent intensity and distribution were measured using IVIS Lumina II in vivo imaging system and Living Image Software (Perkin Elmer) at Day 0, 3 and 7.

\section{Animal model and evaluation}

A total of 15 approximately 6 months old Sprague-Dawley rats (300-350g) were used in this study. OA model was established through the transection of medial meniscotibial ligament (DMM). All rats were randomly divided into 5 groups: (1) Negative control group (rats without surgery receiving weekly saline injection into the knee joint); (2) 
DMM group (OA model rats receiving weekly saline injection into the knee joint); (3)

244 DMM + miR-486-5p group (OA model rats receiving the weekly injection of $20 \mathrm{ul} 10$

$245 \mu \mathrm{g} / \mathrm{mL}$ miR-486-5p mimic in PBS into the knee joint); (4) DMM + miR-486-5p ADSCs

246 group (OA model rats receiving the weekly injection of $20 \mathrm{ul} 1 \times 10^{\wedge} \mathrm{miR}-486-5 \mathrm{p}$ mimic

247 overexpressed ADSCs into the knee joint). (5) DMM + miR-486-5p exos group (OA

248 rats receiving the weekly injection of $20 \mathrm{ul} 10 \mu \mathrm{g} / \mathrm{mL}$ exos from miR-486-5p mimic

249 overexpressed ADSCs into the knee joint). Rats were sacrificed 10 weeks after surgery

250 and the knee joints were harvested for evaluation. Knee joints were fixed, decalcified

251 and imbedded in paraffin and cut into $6 \mu \mathrm{m}$ section. For histological analysis, sections

252 were analyzed using hematoxylin-eosin (HE) staining, safranin-O \& fast green staining.

253 The in vivo apoptosis of chondrocytes was assessed using Terminal

254 deoxynucleotidyl transferse dUTP nick end labelling (TUNEL) assay that was

255 performed according to manufacturer's instructions (C1088, Beyotime). Nuclei were

256 stained with DAPI and apoptotic cells were visualized using Olympus IX51 Inverted

257 Fluorescence Microscope (Olympus).

\section{Statistical analysis}

Data was presented as mean \pm standard deviation (SD). Statistical analysis was

261 performed using SPSS 20.0 software (IBM Corp., NY, USA). The significance of

262 differences between groups was analyzed using Student's t test or one-way ANOVA.

$263 P$ value $<0.05$ was considered significant. 


\section{Results}

\section{Increased inflammation and ER stress in OA synovium and cartilage}

To evaluate the inflammatory and ER stress condition in OA joint, we analyzed the level of several markers in synovium and cartilage. OA synovium demonstrated an increased expression of inflammatory cytokines including IL-1 $\beta$ and TNF- $\alpha$ compared with normal synovium (Fig. 1A). Meanwhile, the expression of proinflammatory M1 macrophage marker iNOS was increased while that of anti-inflammatory M2 macrophage marker CD163 was decreased in OA synovium (Fig. 1A), indicated that synovial macrophage polarized to pro-inflammatory (M1) phenotypes during OA. In

274 line with the inflammatory condition of synovium during OA, an increased level of IL-

$2751 \beta$ and TNF- $\alpha$ was also observed in OA articular cartilage (Fig. 1B). Previous research

276 showed that inflammatory cytokines could lead to prolonged ER stress in chondrocytes

277 (2), indeed more deposition of ER stress markers including CHOP and GRP78 were

278 found in OA articular cartilage compared with normal articular cartilage (Fig. 1B). These

279 results validated that prolonged ER stress may participated in the progression of OA (19).

\section{ER stress participated in IL-1 $\beta$ induced apoptosis of chondrocytes}

283 To investigate the function of ER stress in chondrocyte apoptosis, ER stress inhibitor 4-PBA was used to treat chondrocytes after IL-1 $\beta$ stimulation. The Annexin- $V$ results indicated that IL-1 $\beta$ could induce apoptosis of chondrocyte, which was largely inhibited by ER stress inhibitor 4-PBA (Fig. 2A). The expression of ER stress marker CHOP in 
chondrocytes confirmed that ER stress was elevated after IL-1 $\beta$ stimulation, which could also be downregulated by ER stress inhibitor (Fig. 2B). Western blot further confirmed the involvement of ER stress during IL-1 $1 \beta$ induced apoptosis of chondrocyte.

The expression of ER stress signaling molecules including phosphorylated PERK, 291 phosphorylated IRE1 $\alpha$ and GRP78 were increased over IL-1 $\beta$ stimulation, which could be inhibited by 4-PBA treatment (Fig. 2C). Western blot also indicated that the expression of SOX9, a master transcription factor in chondrogenesis, is also downregulated during IL-1 $\beta$ induced apoptosis (Fig. 2C). This downregulation was also rescued by ER stress inhibition (Fig. 2C). The ELISA results demonstrated that IL-1 $\beta$ could stimulate the secretion of other inflammatory cytokines including IL-6 and TNF$\alpha$ (Fig. 2D), resulting in an inflammatory cascade. In line with declined apoptosis rate, the expression of IL-6 and TNF- $\alpha$ could also be inhibited by 4-PBA treatment (Fig. 2D). These results indicated that ER stress facilitated the inflammation induced apoptosis of chondrocytes.

\section{Characterization of miR-486-5p overexpressing ADSCs and exos}

To investigated the role of miR-486-5p in ADSCs, we transduced miR-486-5p mimic,

NC mimic, miR-486-5p inhibitor or NC inhibitor into normal ADSCs. The expression of miR-486-5p in ADSCs was evaluated using qPCR after transduction. Compared to NC group, the expression of miR-486-5p was significantly up-regulated in miR-486-5p mimic ADSCs group whereas was down-regulated in miR-486-5p inhibitor transduced group (Fig. 3A). After transduction, exos were isolated and purified from culture media 
of each group of ADSCs. qPCR was used to measure the expression of miR-486-5p in five group of exos and a similar trend of miR-486-5p expression was observed as a significant up-regulation in miR-486-5p mimic exos group whereas a significant downregulation in miR-486-5p inhibitor exos group compared to control exos group (Fig. 3B). We further selected two groups of exos we purified to verify their identity. Scanning electron microscope confirmed the round morphology of exos in NC exos and miR486-5p mimic exos groups (Fig. 3C). The mean diameter of exos in NC exos and miR486-5p mimic exos groups were 110.2 and $109.5 \mathrm{~nm}$ as measure by NTA system (Fig. 3D). Western blot analysis suggested positive expression of exos markers like CD9, CD63 and HSP70 in both groups (Fig. 3F). Internalization of exos was confirmed, as PKH26 signal was observed in the perinuclear region of $\mathrm{PKH} 26$-labelled exos treated chondrocytes in both groups (Fig. 3F).

miR-486-5p exos showed better effect than miR-486-5p on attenuating the ER

\section{stress-induced apoptosis in chondrocytes}

To investigate the effect of miR-486-5p on the chondrocyte apoptosis, IL-1 $\beta$ preconditioned chondrocytes were treated with miR-486-5p, NC mimic exos or miR-486$5 p$ mimic exos. Annexin- $V$ results showed that miR-486-5p treatment could

327 significantly inhibit the ER stress induced apoptosis in IL-1 $1 \beta$ pre-conditioned chondrocytes (Fig. 4A). Surprisingly, both NC mimic exos and miR-486-5p mimic exos showed better effect on attenuating the ER stress induced apoptosis in IL-1 $\beta$ preconditioned chondrocytes, whereas miR-486-5p mimic exos showed a trend of lower 
apoptosis rates compared with NC mimic exos (Fig. 4A). The expression of ER stress marker CHOP showed trend of decrement in miR-486-5p group (Fig. 4B). NC mimic exos and miR-486-5p mimic exos both showed greater extent on inhibiting ER stress in chondrocytes than miR-486-5p, among which miR-486-5p mimic exos significantly inhibited the expression of CHOP in IL-1 $\beta$ pre-conditioned chondrocytes (Fig. 4B). Western blot confirmed that apoptosis marker cleaved Caspase-3 and ER stress marker GRP78 showed similar pattern that miR-486-5p mimic exos treatment has the greatest effect on inhibiting apoptosis of chondrocytes (Fig. 4C). miR-486-5p mimic exos treatment also showed the greatest effect on restoring cartilage matrix component type II collagen expression and inhibiting cartilage degradation protease MMP13 expression (Fig. 4C). miR-486-5p mimic exos further reduced the cytokine release of IL- 6 and TNF- $\alpha$ in IL-1 $\beta$ pretreated chondrocyte to the lowest level compared with miR-486-5p and NC exos group (Fig. 4D). These results indicated that miR-486$5 p$ containing exos showed better effect than directly administrated miR-486-5p on attenuating the ER stress-induced apoptosis in chondrocytes.

Exosomal miR-486-5p further improved the effect of exos on attenuating the ER stress-induced apoptosis in chondrocytes

We have demonstrated that both NC mimic Exos and miR-486-5p mimic exos could attenuated the ER stress induced apoptosis (Fig. 4). However, we can't rule out the possibility that this effect was solely induced by exos itself rather than miR-486-5p mimic containing exos. To fully validate the therapeutic effect of miR-486-5p mimic 
exos on attenuating the ER stress induced apoptosis in chondrocytes, we evaluated

the IL-1 $\beta$ pre-conditioned chondrocytes which treated with NC exos, miR-486-5p mimic exos or NC mimic exos. The apoptosis and proliferation rate, ER stress, cartilage matrix related genes and cytokine secretion of chondrocytes were evaluated. As expected, NC exos decreased the apoptosis rate of chondrocytes compared with IL$1 \beta$ group as shown in Annexin- $V$ result (Fig. 5A). EdU results further confirmed the increased proliferation rate over NC exos treatment as the relative EdU incorporation rate was increased (Fig. 5B). However, the further decreased apoptosis rate (Fig. 5A) and the further increased proliferation rate (Fig. 5B) in miR-486-5p exos group suggested the superiority of miR-486-5p exos over NC exos in apoptosis alleviation and proliferation promotion. Meanwhile, the influence of NC exos on ER stress was further investigated using immunofluorescent staining, as $\mathrm{CHOP}$ deposition was decreased by NC exos treatment (Fig. 5C). ER stress was further reduced by miR486-5p exos demonstrated by an even lower level of CHOP in chondrocytes (Fig. 5C). Western blot showed similar results as the expression of cleaved Caspase 3 and GRP78 were downregulated by exos treatments, in which miR-486-5p exos holds the best effect (Fig. 5D). Cartilage matrix deposition and degradation was measured by qPCR and western blot analyses. qPCR result witnessed an increased expression in cartilage matrix deposition markers $A C A N$ and $C O L 2 A 1$, and a decreased expression in matrix degradation markers MMP13 mRNA following NC exos treatment compared with IL-1 $\beta$ group (Fig. 5E). Western blot results further confirmed these conclusions as the expression of type II collagen was increased and MMP13 was decreased 
respectively after NC exos administration (Fig. 5E). The IL-1 $\beta$ induced cartilage matrix gene expression changes were also further rescued by miR-486-5p exos treatment, as evidenced by the increased expression of ACAN, COL2A1 and the decreased expression of MMP13 mRNA (Fig. 5D), as well as the increased type II collagen and decreased protein level of MMP13 in western blot (Fig. 5D). Meanwhile, the inflammatory cytokine secretion profile rescued by NC exos treatment as the concentration of IL-6 and TNF- $\alpha$ were decreased in ELISA data, was further reduced by miR-486-5p exos treatment (Fig. 5F). Taken together, these results showed that miR-486-5p containing exos showed better effect than normal exos on attenuating the ER stress-induced apoptosis in chondrocytes.

\section{The superiority of miR-486-5p exos over miR-486-5p expressing ADSCs in}

\section{attenuation of chondrocyte apoptosis in vitro}

To illustrate the influence of different administrative methods on the attenuation of chondrocyte apoptosis in vitro, miR-486-5p ADSCs and miR-486-5p exos were used to treat IL-1 $\beta$ induced apoptotic chondrocytes. Apparently, both miR-486-5p ADSCs and miR-486-5p exos have inhibitory effect on the apoptosis of chondrocyte as evidenced by the reduced apoptosis rate compared to those in IL-1 $\beta$ group, while miR486-5p exos showed a trend of better effect (Fig. 6A). Meanwhile, the decreased CHOP deposition in both miR-486-5p ADSCs and miR-486-5p exos groups revealed the inhibited ER stress in IL-1 $\beta$ treated chondrocytes, which was decreased to a lower level in miR-486-5p exos group (Fig. 6B). The downregulated expression of cleaved 
Caspase 3 and GRP78 further validated the inhibited apoptosis and ER stress in treatment groups, while miR-486-5p exos showed significantly better effect compared with miR-486-5p ADSCs group (Fig. 6C). Similarly, the increased expression of type II collagen and the decreased expression of MMP13 revealed the remarkably increased chondrogenic matrix deposition in miR-486-5p exos group compared with miR-486-5p ADSCs group (Fig. 6C). Moreover, the increased inflammatory cytokine secretion profile (IL-6 and TNF- $\alpha$ ) in IL-1 $\beta$ group was also reversed after miR-486-5p ADSCs and miR-486-5p exos treatment, with the most significant reversal found in miR-486$5 p$ exos group (Fig. 6D). miR-486-5p containing exos showed better effect than miR486-5p overexpressing ADSCs on attenuating the ER stress-induced apoptosis in chondrocytes.

\section{miR-486-5p tracking in vitro and in vivo}

410 To validated whether the superiority of miR-486-5p mimic exos is due to better delivery, we tracked the sustain of the miR-486-5p under different administrative methods. miR486-5p-Сy3, miR-486-5p-Сy3 mimic ADSCs, and miR-486-5p-Сy3 mimic exos were obtained and used to treat chondrocytes (miR-486-5p-Cy3 mimic ADSCs were added

414 in the upper chamber of Transwell culture system) in non-inflammatory and 415 inflammatory environments $(10 \mathrm{ng} / \mathrm{mL} \mathrm{IL}-1 \beta)$ for $6 \mathrm{~h}, 24 \mathrm{~h}$ and 7 days. Data witnessed 416 a quick uptake of miR-486-5p-Cy3 by chondrocytes in both miR-486-5p-Cy3 and miR417 486-5p-Cy3 exos groups at 6 h (Fig. 7A). After 24 h, the uptake rate in miR-486-5pCy3 group increased, whereas was not long-lasting as it dropped after 7 days while 
that in miR-486-5p-Cy3 exos group kept endurable, especially with inflammatory stimulation (Fig. 7A). However, the fluorescence was barely detected in chondrocytes with miR-486-5p-Cy3 ADSCs administration at $6 \mathrm{~h}$ and $24 \mathrm{~h}$, and a slightly increase of miR-486-5p-Cy3 was witnessed at 7 days even in inflammatory condition (Fig. 7A). In vivo, normal and osteoarthritic knee joint microenvironments (DMM model) were evaluated after the injection of miR-486-5p-Cy3, miR-486-5p-Cy3 ADSCs and miR486-5p-Cy3 exos. The fluorescent intensity was measured using IVIS Lumina II in vivo imaging system at Day 0, 3 and 7. The results showed no obvious diminishment of fluorescence area and intensity in all three groups after 7 days but only the fluorescence was dispersed towards two directions in miR-486-5p-Cy3 ADSCs group (Fig. 7B). Taken together, miR-486-5p-Cy3 exos group seems to be better delivery method.

\section{The superiority of $\mathrm{miR}-486-5 p$ exos in cartilage regeneration and inflammation}

\section{modulation in vivo}

To further investigate the effect of different miR-486-5p mimic administration methods on the cartilage regeneration and inflammation modulation, miR-486-5p, miR-486-5p mimic ADSCs and miR-486-5p mimic exos were injected weekly into the DMM joint, respectively. Cartilage and synovium samples were collected and evaluated 10 weeks after DMM. DMM model was successfully built as evidenced by the disorganized chondrocytes and thinning of cartilage in histological staining (Fig. 8A), and increased apoptotic cells in TUNEL analysis (Fig. 8B). All these changes were significantly 
reversed by miR-486-5p mimic exos and miR-486-5p mimic ADSCs administration, whereas was slightly reversed by miR-486-5p injection (Fig. 8A, B). miR-486-5p mimic exos treatment showed a trend of lower OARSI score and a significantly lower apoptotic cells rate compared with miR-486-5p mimic ADSCs group (Fig. 8A and 8B). Meanwhile, synovium samples exhibited a pro-inflammatory trend as the increased iNOS and decreased CD163 deposition in the DMM group, which was remarkably reversed by miR-486-5p mimic exos injection, slightly reversed with miR-486-5p mimic ADSCs administration and barely changed with miR-486-5p administration (Fig. 8C). These results further validated the superiority of miR-486-5p mimic exos in attenuating chondrocytes apoptosis and OA progression.

\section{Discussion}

Chondrocyte apoptosis is a critical manifestation of the catabolic condition in OA and the inflammatory microenvironment along with ER stress plays an indispensable role in this process. In this study, we demonstrated the therapeutic effect of the miR-486$5 p$ mimic exos in rescuing chondrocytes from the catabolism status in vitro and alleviating OA in vivo.

In response to the inflammatory environmental stress during OA, chondrocytes shift into a catabolic condition that undergoing apoptosis, matrix degradation and inflammatory cytokine secretion, which were found after II-1 $\beta$ administration in this study (20). Extracellular matrix of cartilage is composed of structural proteins and proteoglycans, among which type II collagen is an important structural protein (21). 
The decreased deposition of type II collagen is indicative of ECM dysregulation during OA progression (22). TNF- $\alpha$ and IL-6 are both pro-inflammatory cytokines that play a critical role in inflammatory response (23). Elevated levels of TNF- $\alpha$ and IL-6 have been found in the synovial fluid, suggesting their crucial role in OA pathogenesis (24). Moreover, TNF- $\alpha$ and IL- 6 can induce the production of other cytokines, matrix metalloproteinases (MMPs) and prostaglandins, further inhibit the synthesis of proteoglycans and type II collagen (25). Thus, they play a pivotal role in cartilage matrix degradation and reinforce the vicious cycle in OA (25). Meanwhile, IL-1 1 successfully induced ER stress activation and chondrocyte apoptosis. Inhibiting ER stress resulting in lower apoptosis rate, which is in alignment with previous studies claiming that ER stress participated in the senescence and apoptosis of OA chondrocytes $(2,26)$. ER stress is a double-edged sword as its short term activation induces the unfolded protein response (UPR) to restore homeostasis of chondrocytes, but can cause apoptosis if this response is sustained (27). Apparently in our study, inflammatory microenvironment induced sustained ER stress, which further worsens the catabolic status of chondrocytes as the increased apoptosis rate, matrix deterioration and secretion of inflammatory cytokine.

Meanwhile, we identified miR-486-5p to be an important suppressor in the ER stress-induced chondrocyte apoptosis. Previous studies have reported that direct knee joint administration of miR-140 and miR-26a in OA model reduced cartilage injury and synovitis $(28,29)$. Similarly, in our study, direct administration of miR-486-5p was effective in reversing the catabolic condition of chondrocytes. However, this effect 
turned out to be limited compared to that of miR-486-5p mimic exos. It is not surprising because some MSCs-derived exos are originally abundant in several specific miRNAs and the delivery of these exos was reported to protect articular cartilage in OA mice (30). Meanwhile, exos contain other bioactive molecules that have a synergistic effect with miRNAs. Indeed, our results showed that exos were equipped with better anticatabolic ability with miR-486-5p loading. This coincides with previous studies claiming exosomal miR-9-5p (31) and miR-136-5p (32) from bone marrow-derived MSCs are superior than exos alone in cartilage regeneration. This increased therapeutic potential of miRNA packaging exos can be attributed to the definite suppression of one or several of the downstream target molecule. As according to a previous study, Tob1 inhibition is the key point in the suppression of rheumatoid arthritis by exosomal miR486-5p (33), so Tob1 may be one of the targeted molecule of the miR-486-5p exos we analyzed in this study.

We also explored whether the administration methods affect the efficiency and effectiveness of miR-486-5p. Both MSCs $(34,35)$ and their exos $(15,36)$ are candidates for OA therapy. Exos possess more advantages over MSCs including convenience in storage, stable biological activity, low risk of iatrogenic tumor formation and minimal immunogenicity (37). Previous studies comparing the therapeutic effect on OA and found that exos were superior than their originated MSCs (38). In our study, exos were also found to be a better carrier than ADSCs in miR-486-5p administration for apoptosis alleviation and matrix regeneration of chondrocytes. This result is a little astonishing because we originally believed that miR-486-5p mimic ADSCs are 
supposed to secrete miR-486-5p mimic exos. However, the limited miR-486-5p signal in the miR-486-5p mimic ADSCs group chondrocytes is probably due to the poor release of miR-486-5p, as inflammation tends to have a significant impact on the secretome of ADSCs (39).

511 All our in vitro results were mirrored in the in vivo study. Firstly, compared with miR486-5p alone, the administration of miR-486-5p mimic ADSCs resulted in decreased

513 cartilage erosion. Indeed our in vivo imaging demonstrated a polarized diffusion signal

5147 days after injection, which suggested the migration of ADSCs onto the injured cartilage, as MSCs tend to accumulate in damaged tissue and provides a possible explanation for the superior results for in situ remediation (40). Exos also have a target-

517 homing character (40). Originally, we thought that the administration of MSCs should generate a similar, or even better effect on apoptosis prevention and matrix regeneration because MSCs are supposed to have exosome secretion as well as chondrogenic differentiation and immunoregulatory effect after joint injection. However,

521 our data witnessed an increased deposition of chondroitin sulfate following miR-486-

$5225 p$ mimic exos instead of miR-486-5p mimic ADSCs administration. Similarly, TUNEL 523 data confirmed that an almost vanished deposition of apoptosis signal in both cartilage 524 and subchondral layer with miR-486-5p mimic exos administration, whereas miR-486-

$5255 p$ mimic ADSCs showed lesser effect to rescue the chondrocyte apoptosis in the 526 cartilage layer. We speculated that the potential reason is the rapid elimination of 527 MSCs after joint administration and even if they survived, rarely was there 528 differentiation into the desired cells $(41,42)$. 

the pro-inflammatory M1 and the anti-inflammatory M2 phenotypes. Accumulating

531 evidence suggests that the synovial inflammation is correlated with the pathogenesis

532 and progression of OA (43), and research showed that clinical symptoms of OA are

533 correlated with synovial inflammation rather than structural pathology (44). MSCs are

534 susceptive to certain "environmentally responsive" function in the microenvironment

535 (45). Apart from secretion factors, synovial M1 macrophages inhibit chondrogenesis of

536 stem cells while M2 macrophages support the survival of cartilage grafts. In most

537 conditions, MSCs have an anti-inflammatory role to suppress the activation of M1

538 macrophages and promote M2 polarization in vitro (46). However, low levels of

539 inflammatory stimuli can endow MSCs with a pro-inflammatory mode and influence the

540 quality of their secretome (39), which further suggests the necessity of acquiring the

541 secretion products of MSCs before putting in the adverse circumstances. Recent

542 publications have indicated that the anti-inflammatory effects of MSC-derived exos

543 were relied on the transportation of immunoregulatory miRNAs and proteins into

544 inflammatory immune cells, inhibiting the generation of M1 phenotype macrophages

545 and enabling their phenotypic transformation into immunosuppressive M2

546 macrophages (37). Notably, they have demonstrated the role of ADSC-derived exos

547 on macrophages polarization. Similarly, in our study, treatment with miR-486-5p mimic

548 exos promotes M2 macrophages polarization, contributing to the attenuation of the on-

549 going inflammation, and creates a favorable environment for cartilage regeneration.

550 Interestingly, miR-486-5p mimic exos demonstrated a remarkably increased diffusion 

uptake by other cell types including synovial cells, which induce widespread anti-

553 inflammation in the joint.

\section{Conclusions}

Taken together, these results support the therapeutic role of miR-486-5p mimic exos in inhibiting chondrocytes apoptosis in vitro and alleviating OA in vivo. Despite the superiority over miR-486-5p and miR-486-5p mimic ADSCs, more critical technological considerations including the route and dose of miR-486-5p mimic exos need to be further explored before clinical applications.

\section{Abbreviations}

OA: osteoarthritis; ER: endoplasmic reticulum; miRNAs: microRNAs; ADSCs: adipose derived stem cells; MSCs: mesenchymal stem cells; exos: exosomes; DMM: destabilization of the medial meniscus; TUNEL: terminal deoxynucleotidyl transferse dUTP nick end labelling.

\section{Acknowledgements}

569 The authors would like to thank Translational Medicine Center of the Shanghai East Hospital of Tongji University for help with this study.

\section{Authors' contributions}


573 YW: chondrocytes experiments, data collection and manuscript writing. AF: animal

574 experiments, data analysis and manuscript writing. ZL and LY: human samples

575 collection. LL: hADSCs isolation. ZP, MM and SL: exosomes characterization and

576 isolation. JC and FY: conception and design of the study, and manuscript revising. All

577 authors read and approved the final version of the manuscript.

\section{Funding}

580 This project was sponsored by grants from Shanghai Sailing Program (20YF1440500),

581 the Ministry of Science and Technology of China (2020YFC2002800) and National

582 Natural Science Foundation of China (82002297).

\section{Availability of data and materials}

585 The data that support the findings of this study are available from the corresponding 586 author upon reasonable request.

\section{Declarations}

\section{Ethics approval and consent to participate}

The study was conducted according to the guidelines of the Institutional Review

591 Boards at Shanghai East Hospital.

\section{Consent for publication}

Not applicable. 


\section{Competing interests}

597 The authors declare that they have no competing interests.

\section{References}

1. Robinson $W H$, Lepus $C M$, Wang $Q$, Raghu $H$, Mao R, Lindstrom TM, et al. Low-

601 grade inflammation as a key mediator of the pathogenesis of osteoarthritis. Nature

602 reviews Rheumatology. 2016;12(10):580-92.

2. Liu Y, Zhu H, Yan X, Gu H, Gu Z, Liu F. Endoplasmic reticulum stress participates in the progress of senescence and apoptosis of osteoarthritis chondrocytes. Biochem Biophys Res Commun. 2017;491(2):368-73.

3. Liu L, Yan LN, Sui Z. MicroRNA-150 affects endoplasmic reticulum stress via MALAT1-miR-150 axis-mediated NF-kappaB pathway in LPS-challenged HUVECs and septic mice. Life Sci. 2021;265:118744.

4. Kassan M, Vikram A, Li Q, Kim YR, Kumar S, Gabani M, et al. MicroRNA-204 promotes vascular endoplasmic reticulum stress and endothelial dysfunction by targeting Sirtuin1. Sci Rep. 2017;7(1):9308.

5. Li H, Li Z, Pi Y, Chen Y, Mei L, Luo Y, et al. MicroRNA-375 exacerbates knee osteoarthritis through repressing chondrocyte autophagy by targeting ATG2B. Aging (Albany NY). 2020;12(8):7248-61. marker in cervical cancer: with investigation of potential mechanisms. BMC Cancer. 

biomarker in cancer diagnosis and prognosis: a systematic review and meta-analysis. Oncotarget. 2018;9(17):13948-58. miRNAs and their target genes involved in cell apoptosis during intervertebral disc degeneration development using bioinformatics methods. J Neurosurg Sci. 2020.

9. Kong R, Gao J, Si Y, Zhao D. Combination of circulating miR-19b-3p, miR-122-5p and miR-486-5p expressions correlates with risk and disease severity of knee osteoarthritis. Am J TransI Res. 2017;9(6):2852-64. 10. Ouboussad L, Hunt L, Hensor EMA, Nam JL, Barnes NA, Emery P, et al. Profiling microRNAs in individuals at risk of progression to rheumatoid arthritis. Arthritis Res Ther. 2017;19(1):288.

11. Chai X, Si H, Song J, Chong Y, Wang J, Zhao G. miR-486-5p Inhibits Inflammatory Response, Matrix Degradation and Apoptosis of Nucleus Pulposus Cells through Directly Targeting FOXO1 in Intervertebral Disc Degeneration. Cell Physiol Biochem. 2019;52(1):109-18.

12. Maheshwari R, Tekade M, Gondaliya P, Kalia K, D'Emanuele A, Tekade RK. Recent advances in exosome-based nanovehicles as RNA interference therapeutic carriers. Nanomedicine (Lond). 2017;12(21):2653-75. 13. Toh WS, Lai RC, Hui JHP, Lim SK. MSC exosome as a cell-free MSC therapy for cartilage regeneration: Implications for osteoarthritis treatment. Seminars in cell \& 
developmental biology. 2017;67:56-64.

14. Stranford DM, Leonard JN. Delivery of Biomolecules via Extracellular Vesicles: A Budding Therapeutic Strategy. Adv Genet. 2017;98:155-75.

15. Zhang S, Chuah SJ, Lai RC, Hui JHP, Lim SK, Toh WS. MSC exosomes mediate cartilage repair by enhancing proliferation, attenuating apoptosis and modulating immune reactivity. Biomaterials. 2018;156:16-27.

16. Zhang S, Chu WC, Lai RC, Lim SK, Hui JH, Toh WS. Exosomes derived from human embryonic mesenchymal stem cells promote osteochondral regeneration. Osteoarthritis and cartilage. 2016;24(12):2135-40.

17. Mao G, Zhang Z, Hu S, Zhang Z, Chang Z, Huang Z, et al. Exosomes derived from miR-92a-3p-overexpressing human mesenchymal stem cells enhance chondrogenesis and suppress cartilage degradation via targeting WNT5A. Stem Cell Res Ther. 2018;9(1):247.

18. Tao SC, Yuan T, Zhang YL, Yin WJ, Guo SC, Zhang CQ. Exosomes derived from miR-140-5p-overexpressing human synovial mesenchymal stem cells enhance cartilage tissue regeneration and prevent osteoarthritis of the knee in a rat model. Theranostics. 2017;7(1):180-95.

19. Hughes A, Oxford AE, Tawara K, Jorcyk CL, Oxford JT. Endoplasmic Reticulum Stress and Unfolded Protein Response in Cartilage Pathophysiology; Contributing Factors to Apoptosis and Osteoarthritis. Int J Mol Sci. 2017;18(3).

20. Zheng L, Zhang Z, Sheng P, Mobasheri A. The role of metabolism in chondrocyte dysfunction and the progression of osteoarthritis. Ageing Res Rev. 2021;66:101249. 
661

662

663

664

665

666

667

668

669

670

671

672

673

674

675

676

677

679

680

681

682

21. Lefebvre V, Angelozzi M, Haseeb A. SOX9 in cartilage development and disease.

Curr Opin Cell Biol. 2019;61:39-47.

22. Rahmati M, Nalesso G, Mobasheri A, Mozafari M. Aging and osteoarthritis: Central role of the extracellular matrix. Ageing Res Rev. 2017;40:20-30.

23. Wang T, He C. TNF-alpha and IL-6: The Link between Immune and Bone System. Curr Drug Targets. 2020;21(3):213-27.

24. Kapoor M, Martel-Pelletier J, Lajeunesse D, Pelletier JP, Fahmi H. Role of proinflammatory cytokines in the pathophysiology of osteoarthritis. Nature reviews Rheumatology. 2011;7(1):33-42.

25. Wang T, He C. Pro-inflammatory cytokines: The link between obesity and osteoarthritis. Cytokine Growth Factor Rev. 2018;44:38-50.

26. Uehara Y, Hirose J, Yamabe S, Okamoto N, Okada T, Oyadomari S, et al. Endoplasmic reticulum stress-induced apoptosis contributes to articular cartilage degeneration via C/EBP homologous protein. Osteoarthritis Cartilage. $2014 ; 22(7): 1007-17$

27. Hetz $\mathrm{C}$. The unfolded protein response: controlling cell fate decisions under ER stress and beyond. Nat Rev Mol Cell Biol. 2012;13(2):89-102.

28. Zhao Z, Dai XS, Wang ZY, Bao ZQ, Guan JZ. MicroRNA-26a reduces synovial inflammation and cartilage injury in osteoarthritis of knee joints through impairing the NF-kappaB signaling pathway. Biosci Rep. 2019;39(4).

29. Si HB, Zeng Y, Liu SY, Zhou ZK, Chen YN, Cheng JQ, et al. Intra-articular injection of microRNA-140 (miRNA-140) alleviates osteoarthritis (OA) progression by 
683

684

685

686

687

688

689

690

691

692

693

694

695

696

697

698

699

700

701

702

703

704

modulating extracellular matrix (ECM) homeostasis in rats. Osteoarthritis Cartilage. 2017;25(10):1698-707 .

30. Wu J, Kuang L, Chen C, Yang J, Zeng WN, Li T, et al. miR-100-5p-abundant exosomes derived from infrapatellar fat pad MSCs protect articular cartilage and ameliorate gait abnormalities via inhibition of mTOR in osteoarthritis. Biomaterials. 2019;206:87-100.

31. Jin Z, Ren J, Qi S. Exosomal miR-9-5p secreted by bone marrow-derived mesenchymal stem cells alleviates osteoarthritis by inhibiting syndecan-1. Cell Tissue Res. 2020;381(1):99-114.

32. Chen X, Shi Y, Xue P, Ma X, Li J, Zhang J. Mesenchymal stem cell-derived exosomal microRNA-136-5p inhibits chondrocyte degeneration in traumatic osteoarthritis by targeting ELF3. Arthritis Res Ther. 2020;22(1):256.

33. Chen J, Liu M, Luo X, Peng L, Zhao Z, He C, et al. Exosomal miRNA-486-5p derived from rheumatoid arthritis fibroblast-like synoviocytes induces osteoblast differentiation through the Tob1/BMP/Smad pathway. Biomater Sci. 2020;8(12):343042.

34. Matas J, Orrego M, Amenabar D, Infante C, Tapia-Limonchi R, Cadiz Ml, et al. Umbilical Cord-Derived Mesenchymal Stromal Cells (MSCs) for Knee Osteoarthritis: Repeated MSC Dosing Is Superior to a Single MSC Dose and to Hyaluronic Acid in a Controlled Randomized Phase I/II Trial. Stem cells translational medicine. 2019;8(3):215-24 .

35. Barry F. MSC Therapy for Osteoarthritis: An Unfinished Story. J Orthop Res. 
2019;37(6):1229-35.

36. Zhang S, Teo KYW, Chuah SJ, Lai RC, Lim SK, Toh WS. MSC exosomes alleviate temporomandibular joint osteoarthritis by attenuating inflammation and restoring matrix homeostasis. Biomaterials. 2019;200:35-47.

37. Harrell CR, Fellabaum C, Jovicic N, Djonov V, Arsenijevic N, Volarevic V. Molecular Mechanisms Responsible for Therapeutic Potential of Mesenchymal Stem CellDerived Secretome. Cells. 2019;8(5).

38. Zavatti M, Beretti F, Casciaro F, Bertucci E, Maraldi T. Comparison of the therapeutic effect of amniotic fluid stem cells and their exosomes on monoiodoacetateinduced animal model of osteoarthritis. Biofactors. 2020;46(1):106-17.

39. Lee MJ, Kim J, Kim MY, Bae YS, Ryu SH, Lee TG, et al. Proteomic analysis of tumor necrosis factor-alpha-induced secretome of human adipose tissue-derived mesenchymal stem cells. J Proteome Res. 2010;9(4):1754-62.

40. Shi Y, Wang Y, Li Q, Liu K, Hou J, Shao C, et al. Immunoregulatory mechanisms of mesenchymal stem and stromal cells in inflammatory diseases. Nat Rev Nephrol. 2018;14(8):493-507.

41. Wyles CC, Houdek MT, Behfar A, Sierra RJ. Mesenchymal stem cell therapy for osteoarthritis: current perspectives. Stem Cells Cloning. 2015;8:117-24.

42. Liu S, Liu D, Chen C, Hamamura K, Moshaverinia A, Yang R, et al. MSC Transplantation Improves Osteopenia via Epigenetic Regulation of Notch Signaling in Lupus. Cell Metab. 2015;22(4):606-18.

43. Zhang $\mathrm{H}$, Lin $\mathrm{C}$, Zeng $\mathrm{C}$, Wang $\mathrm{Z}$, Wang $\mathrm{H}$, Lu J, et al. Synovial macrophage M1 
polarisation exacerbates experimental osteoarthritis partially through R-spondin-2.

Ann Rheum Dis. 2018;77(10):1524-34.

44. Shu CC, Zaki S, Ravi V, Schiavinato A, Smith MM, Little CB. The relationship between synovial inflammation, structural pathology, and pain in post-traumatic osteoarthritis: differential effect of stem cell and hyaluronan treatment. Arthritis Res Ther. 2020;22(1):29.

45. Murphy MB, Moncivais K, Caplan Al. Mesenchymal stem cells: environmentally responsive therapeutics for regenerative medicine. Exp Mol Med. 2013;45:e54.

46. Harrell CR, Markovic BS, Fellabaum C, Arsenijevic A, Volarevic V. Mesenchymal stem cell-based therapy of osteoarthritis: Current knowledge and future perspectives.

\section{Biomed Pharmacother. 2019;109:2318-26.}

Figure legends

A
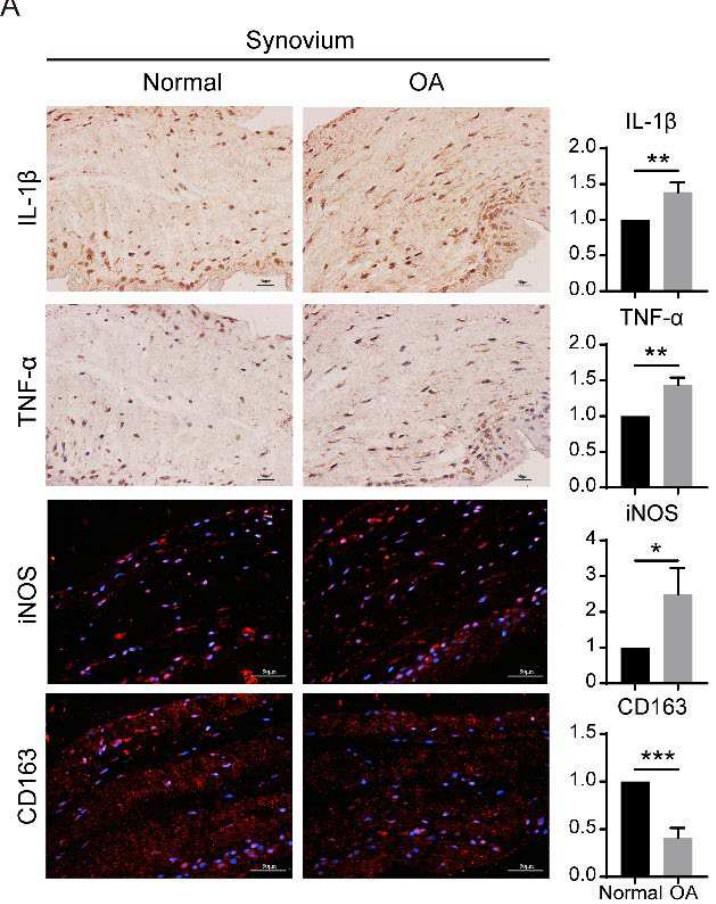

B

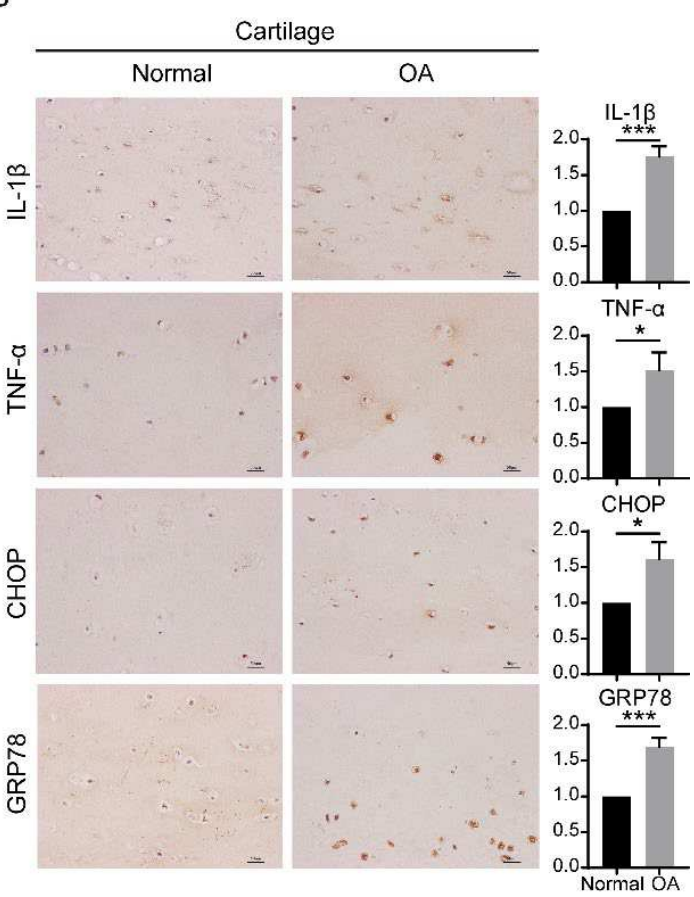

Fig. 1 Evaluation of inflammation and ER stress in normal and OA synovium and 
cartilage. (A) Immunohistochemical and immunofluorescent staining of inflammatory cytokines IL-1 $\beta$ and TNF- $\alpha$, M1 macrophage marker iNOS and M2 macrophage marker

744 CD163 in normal and OA synovium samples. (B) Immunohistochemical staining of IL-

$7451 \beta$, TNF- $\alpha$ and ER stress markers CHOP and GRP78 in normal and OA articular cartilage samples. The statistical significance was assessed using two-tailed Student's unpaired $t$ tests. Data represent mean $\pm \operatorname{SD}\left({ }^{*} p<0.05,{ }^{* *} p<0.01,{ }^{* * *} p<0.001\right)$. 
A

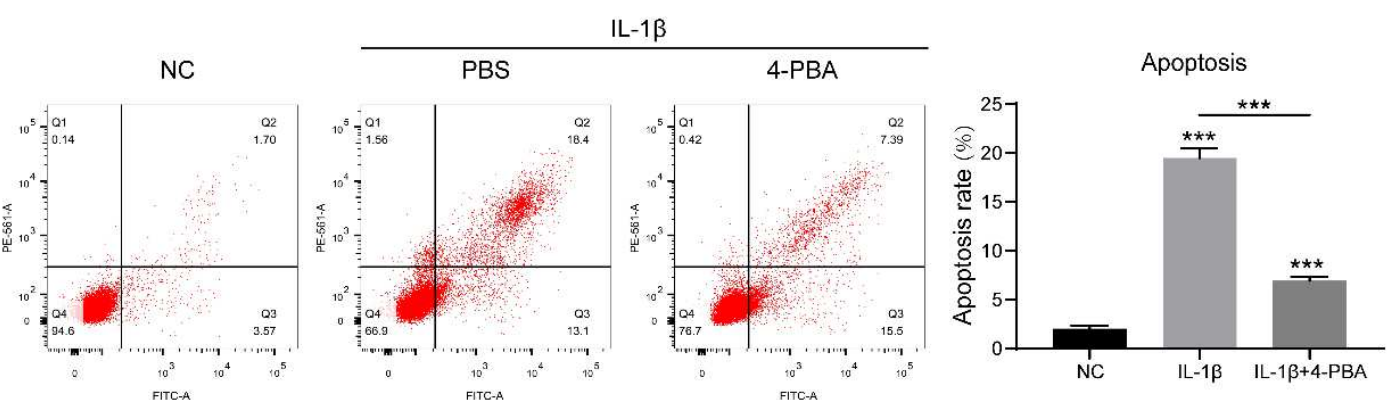

B
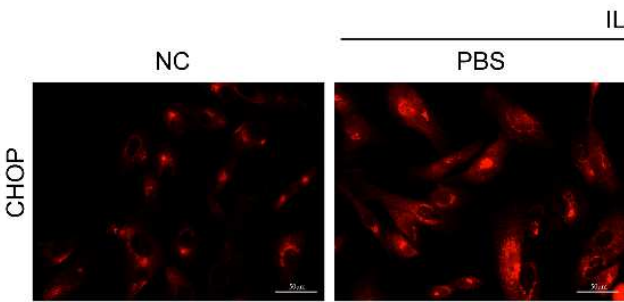

IL-1 $1 \beta$
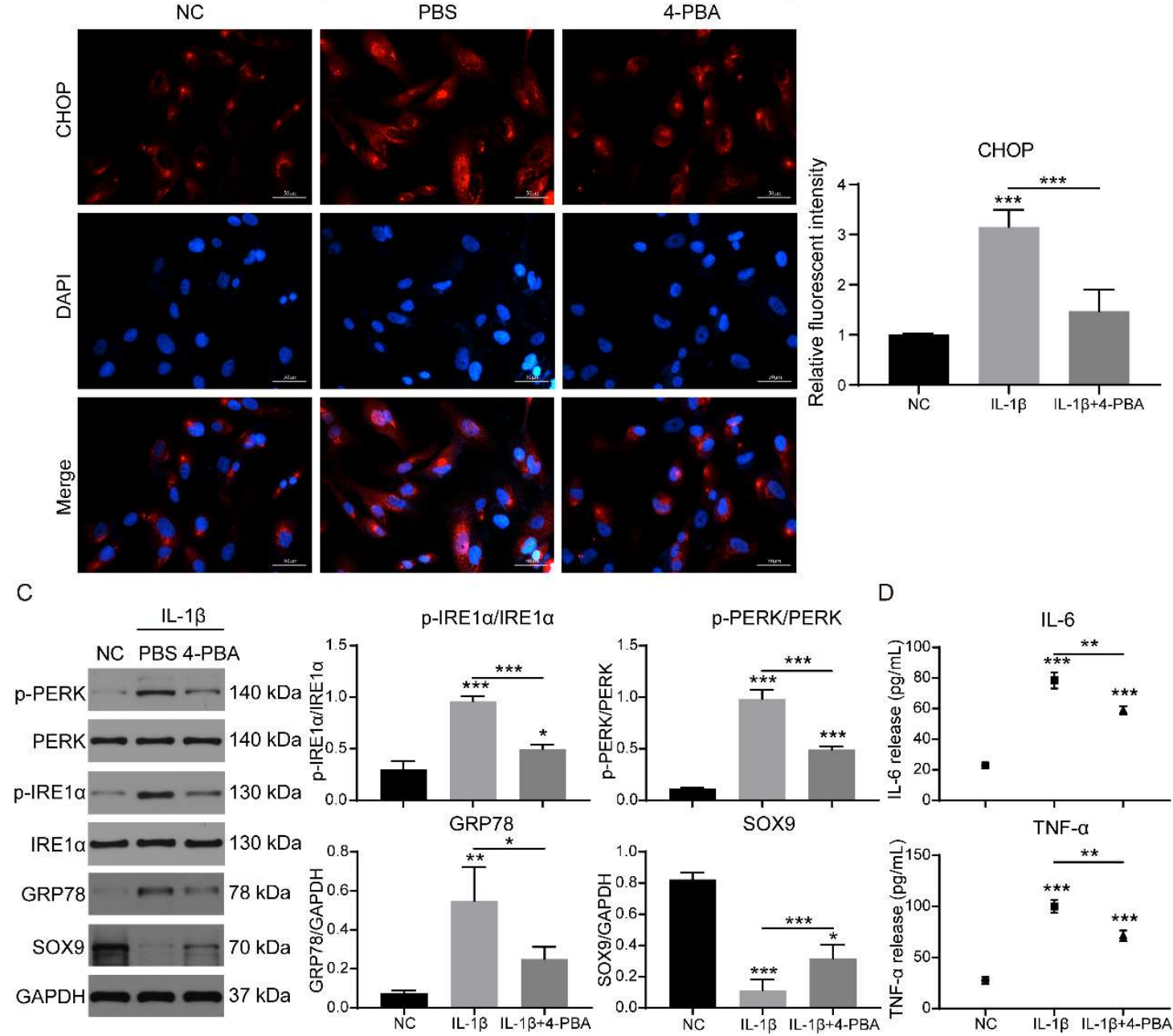

Fig. 2 Role of ER stress on IL-1 $\beta$-induced apoptosis of chondrocytes.

Chondrocytes (NC group) were treated with $10 \mathrm{ng} / \mathrm{mL}$ IL-1 $\beta$ (IL-1 $\beta$ group) or IL-1 $\beta+$

751 ER stress inhibitor 4-PBA (4-PBA group). (A) The apoptosis rate was evaluated using

Annexin-V Staining.

(B) The expression of CHOP was evaluated using

753 immunofluorescent staining. (C) The expression of p-PERK, PERK, p-IRE1 $\alpha$, IRE1 $\alpha$, 
D

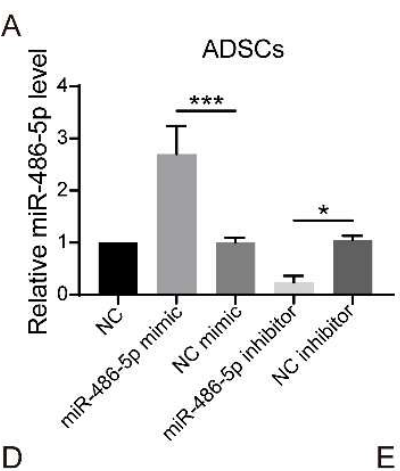

E

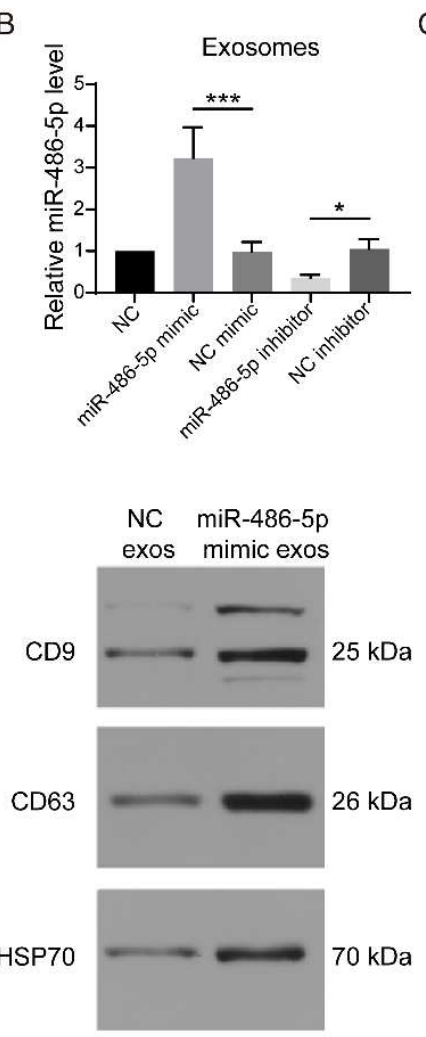

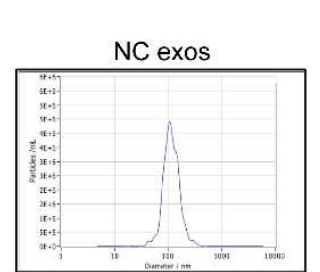

miR-486-5p mimic exos

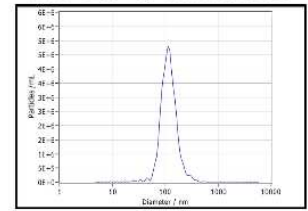

C
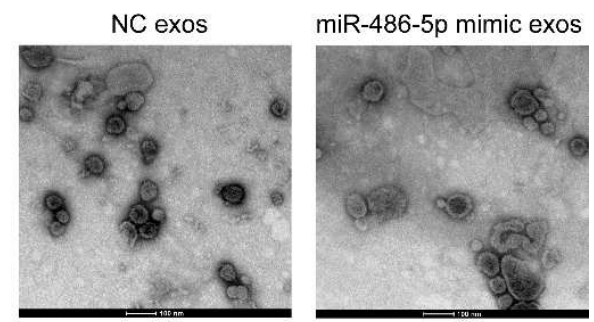

F

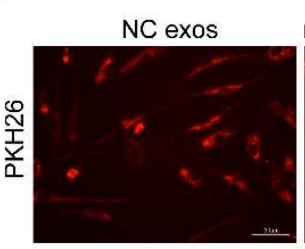

miR-486-5p mimic exos
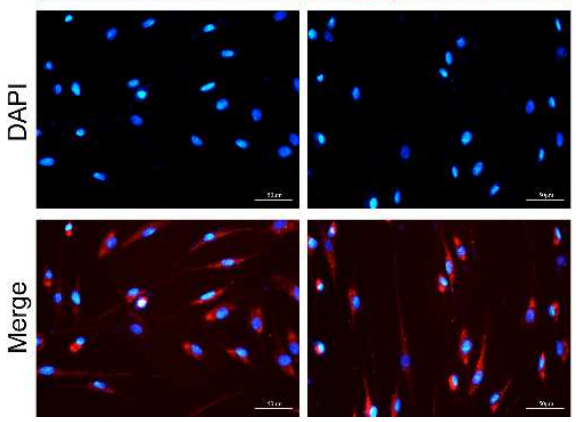

760

Fig. 3 Characterization of miR-486-5p transfected ADSCs and exos. miR-486-5p

mimic, negative control mimic (NC mimic), miR-486-5p inhibitor and NC inhibitor were transfected into normal ADSCs (NC group) before exos were obtained from the corresponding group of ADSCs. The expression of miR-486-5p in ADSCs (A) and the corresponding exos (B) were evaluated. (C) Scanning Electron Microscope was used to observe exos from NC group ADSCs (NC exos) and miR-486-5p mimic transfected ADSCs (miR-486-5p exos). (D) The diameter of exos was determined using Nanoparticle Tracking Analysis (NTA). (E) The expression of exosome markers CD9, 
771 tests. Data represent mean $\pm \operatorname{SD}\left({ }^{*} p<0.05,{ }^{* * *} p<0.001\right)$.

A A
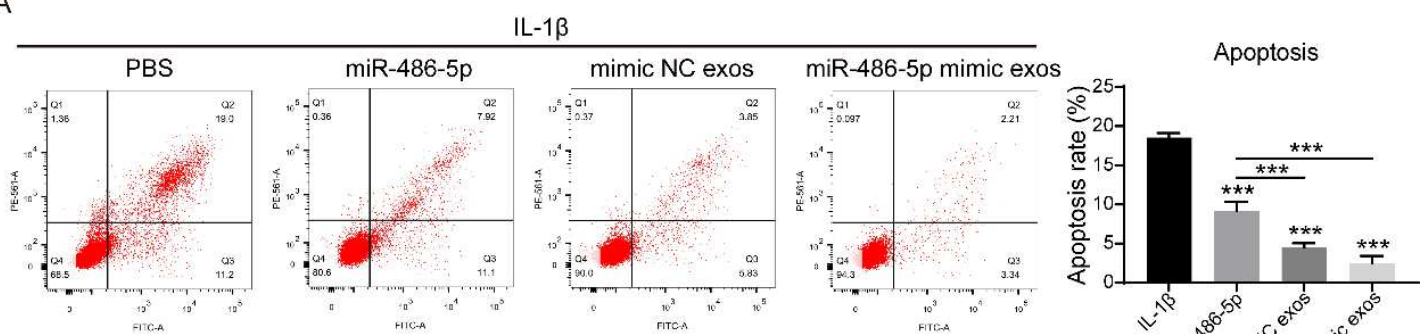

B
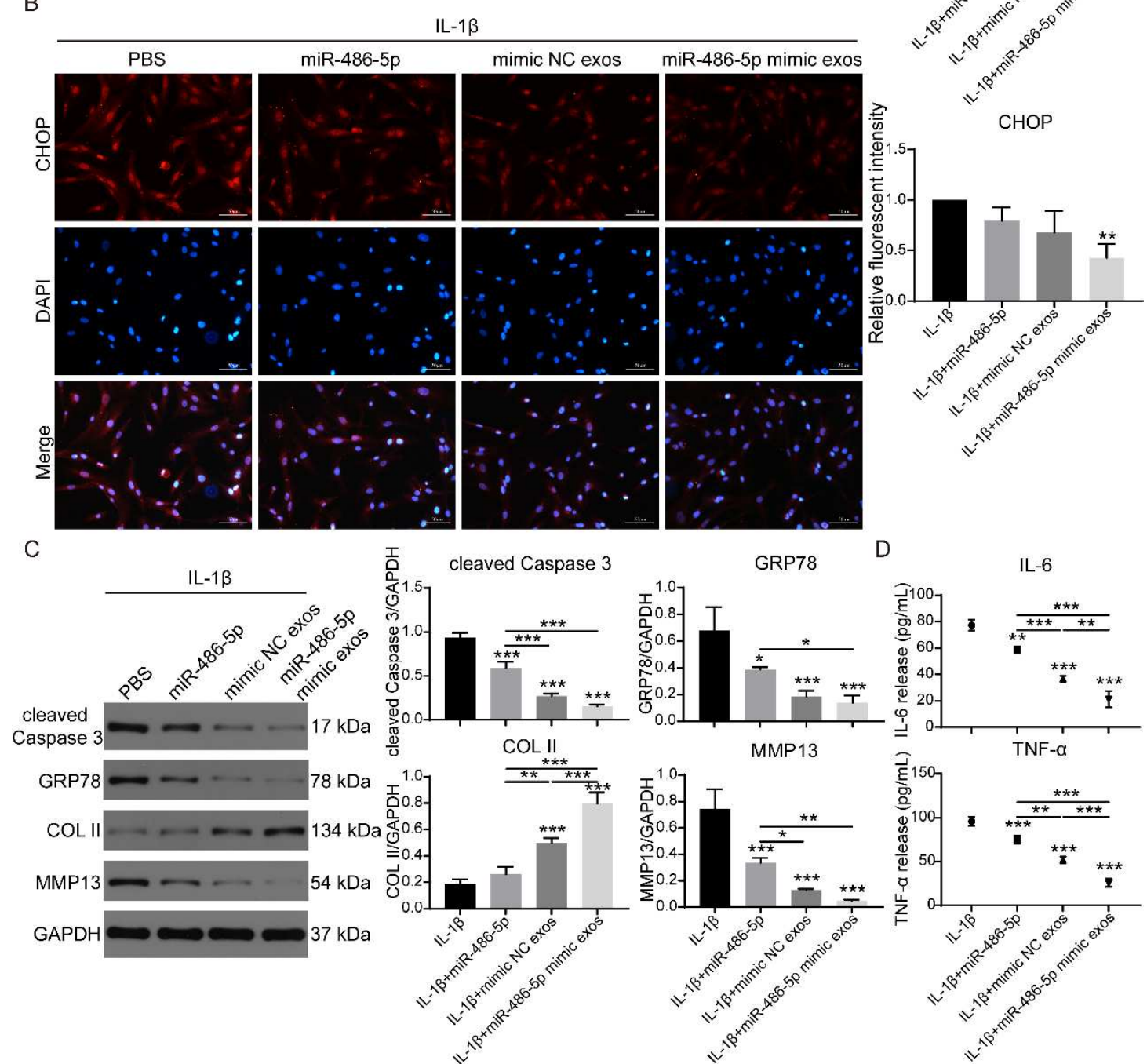

Fig. 4 Comparation of miR-486-5p and exosomal miR-486-5p on ER stress and

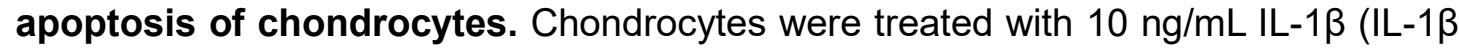


775 group), IL-1 $\beta+$ miR-486-5p mimic (miR-486-5p group), IL-1 $\beta+$ exos from miR-486-5p

776 mimic NC transfected ADSCs (miR-486-5p NC exos group) and IL-1 $\beta+$ exos from

777 miR-486-5p mimic transfected ADSCs (miR-486-5p exos group). (A) The apoptosis

778 rate of each group was evaluated using Annexin-V Staining. (B) The expression of

779 CHOP of each group was evaluated using immunofluorescent staining. (C) The

780 expression of cleaved Caspase-3, GRP78, type II collagen and MMP-13 were

781 evaluated using Western Blot. GAPDH served as internal control. (D) The

782 concentration of IL-6 and TNF- $\alpha$ were evaluated using ELISA. The statistical

783 significance was assessed using one-way ANOVAs with Tukey's multiple comparison

784 tests. Data represent mean $\pm \mathrm{SD}\left({ }^{*} p<0.05,{ }^{* *} p<0.01,{ }^{* * *} p<0.001\right)$. 
A

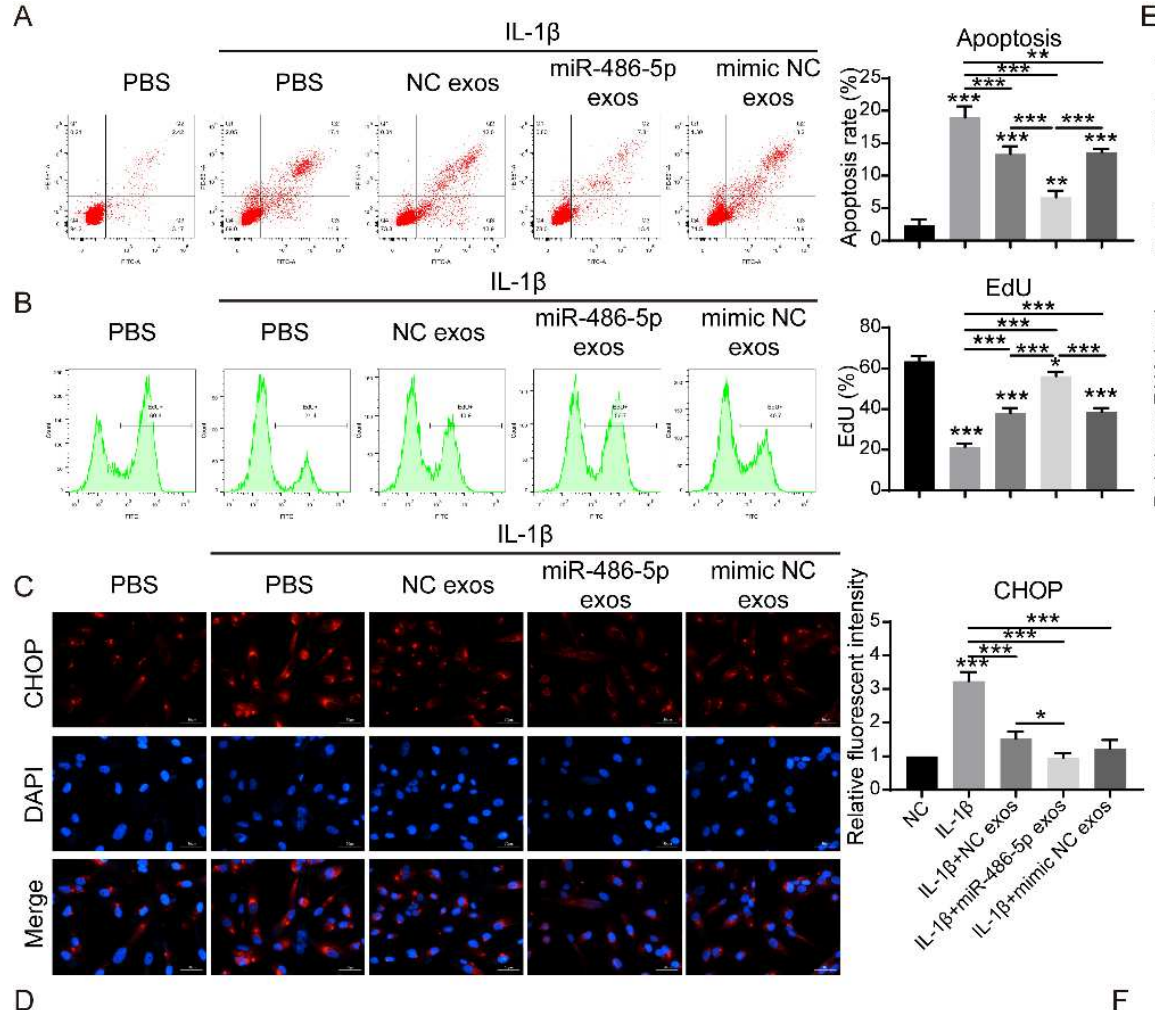

$\mathrm{E}$
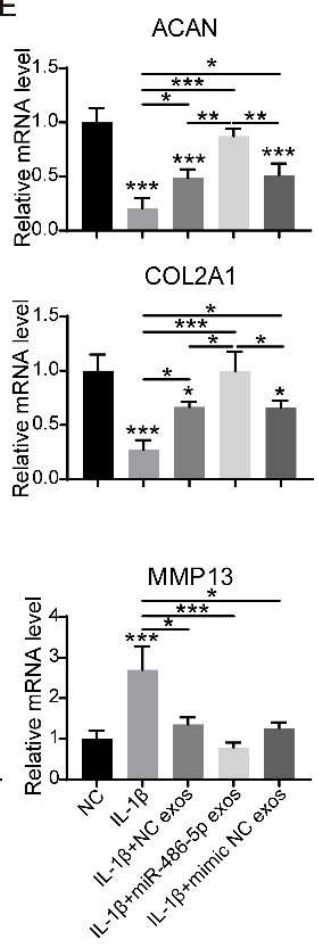

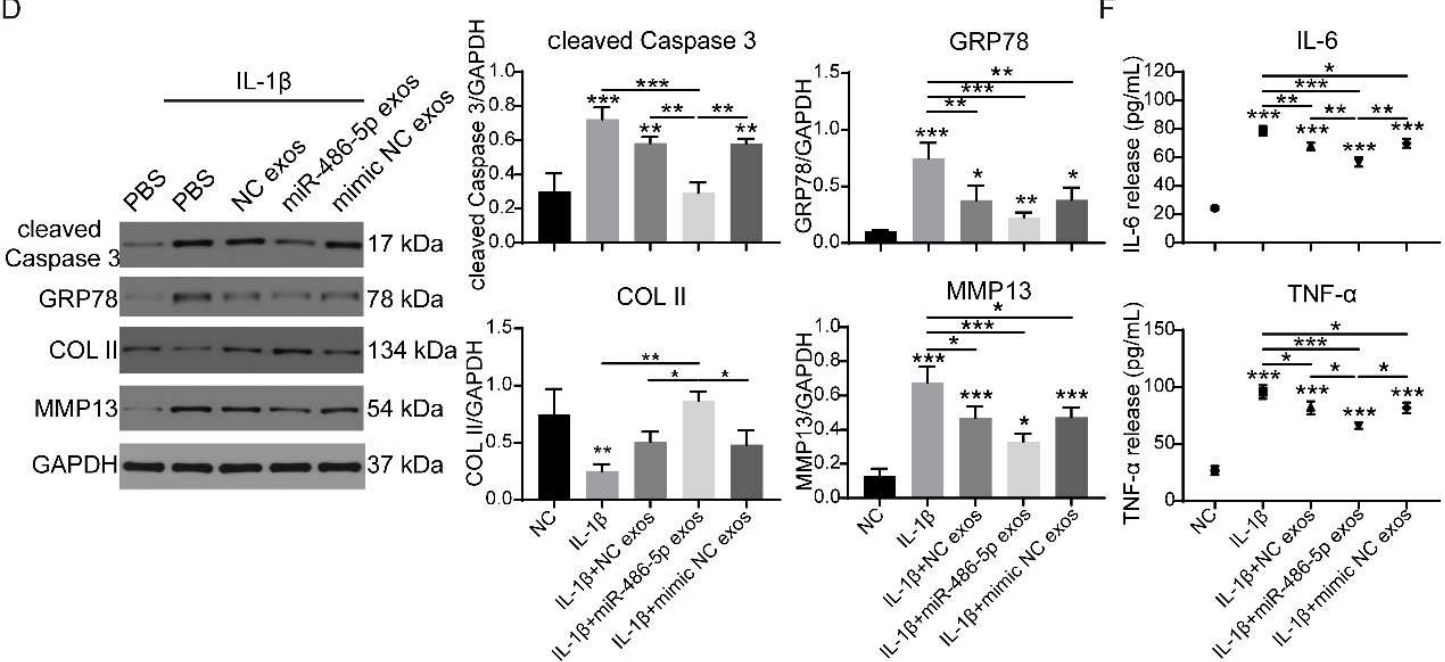

Fig. 5 Comparation of exosomes and miR-486-5p packaging exosomes on ER exos from miR-486-5p mimic transfected ADSCs (miR-486-5p exos group) and IL-1 $\beta$ + exos from miR-486-5p mimic NC transfected ADSCs (miR-486-5p NC exos group). (A) The apoptosis rate of each group was evaluated using Annexin-V staining. (B) The proliferation rate of each group was evaluated using Click-i ${ }^{\circledR}$ EdU assay. (C) The 
expression of CHOP was evaluated using immunofluorescent staining. (D) The

794 expression of cleaved Caspase-3, GRP78, type II collagen and MMP-13 were

795 evaluated using Western Blot. GAPDH served as internal control. (E) The expression

796 of mRNA ACAN, COL2A1 and MMP13 of each group were evaluated. (F) The

797 concentration of IL-6 and TNF- $\alpha$ in culture media were evaluated using ELISA. The

798 statistical significance was assessed using one-way ANOVAs with Tukey's multiple

799 comparison tests. Data represent mean $\pm \operatorname{SD}\left({ }^{*} p<0.05,{ }^{* *} p<0.01,{ }^{* * *} p<0.001\right)$.

A

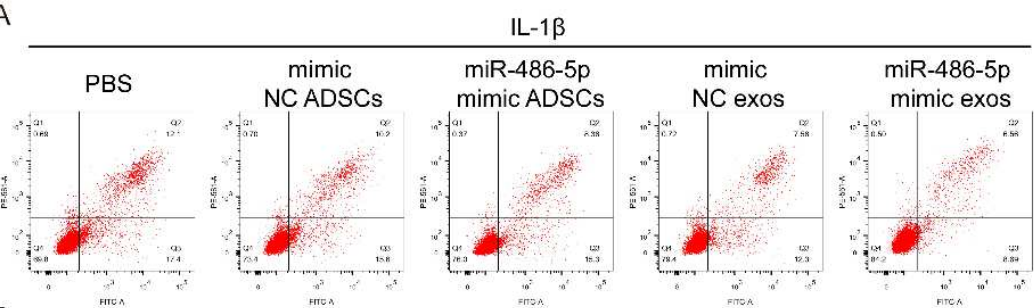

B
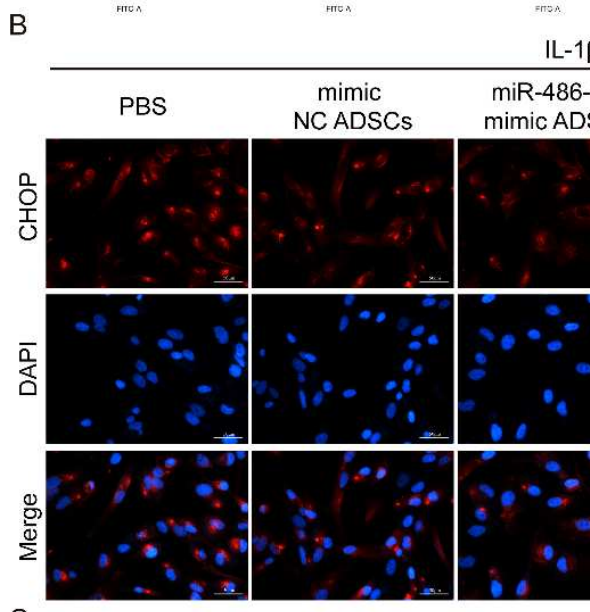

C

IL-1 $1 \beta$

mimic ADSCs
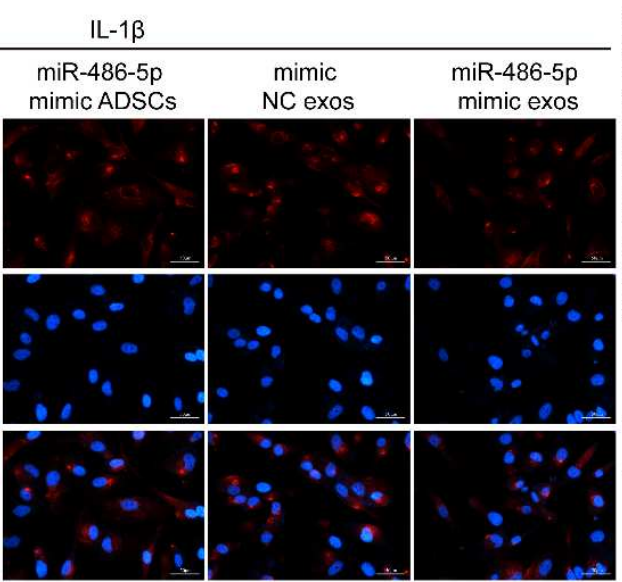
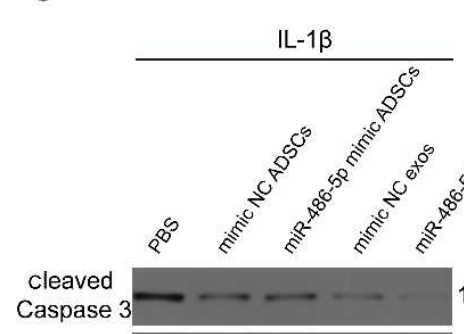

GRP78

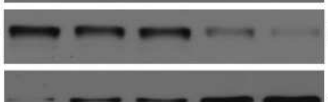

COL I
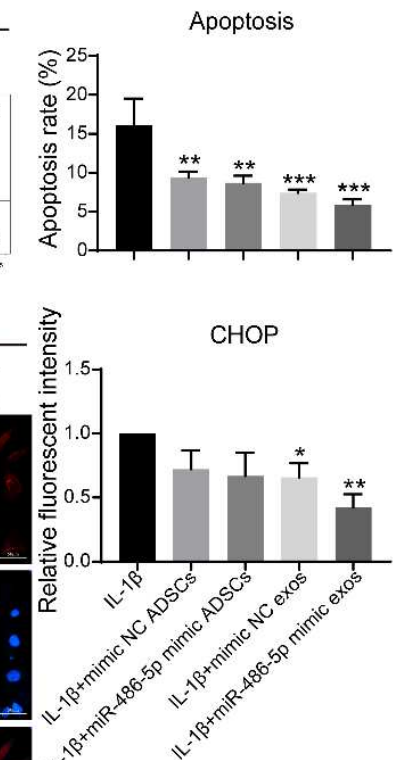

MMP13

$--54 \mathrm{kDa}$
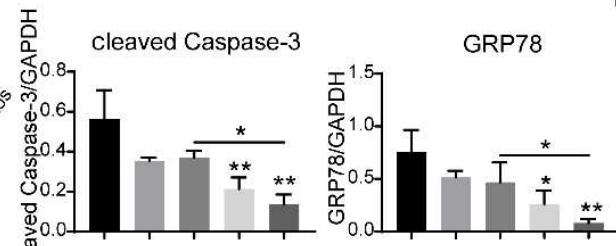

D

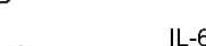

GAPDH

$37 \mathrm{kDa}$
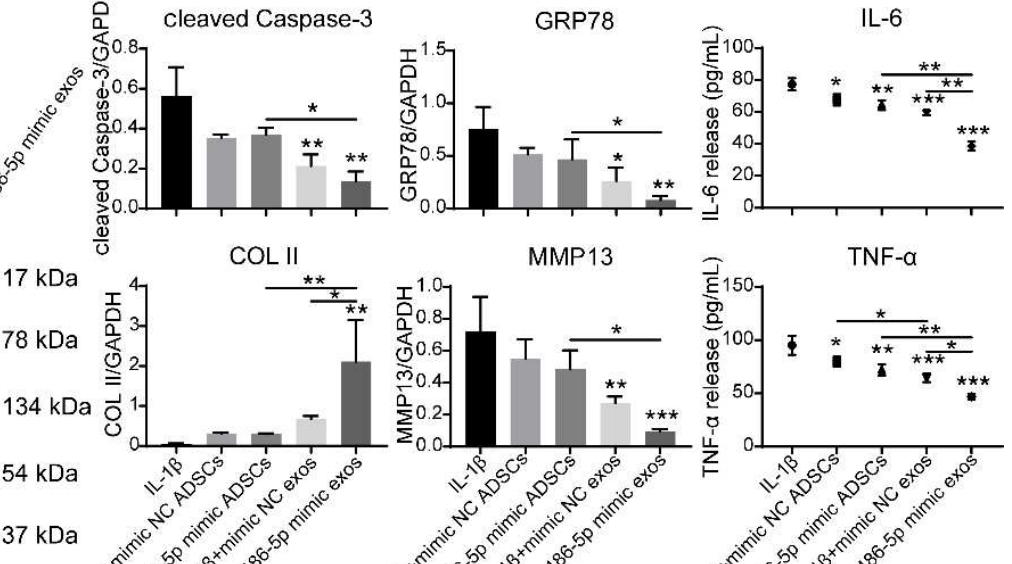
Da
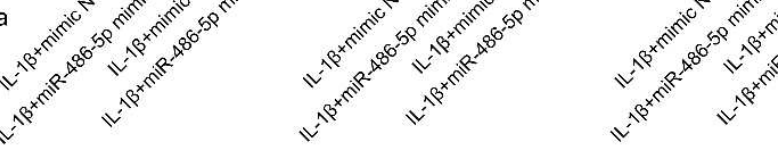

ADSCs on ER stress and apoptosis of chondrocytes. Chondrocyte were treated with $10 \mathrm{ng} / \mathrm{mL}$ IL-1 $($ IL-1 $\beta$ group), IL-1 $\beta+$ miR-486-5p mimic NC transfected ADSCs

804 (mimic NC ADSCs group), IL-1 $\beta+$ miR-486-5p mimic transfected ADSCs (miR-486-5p 805 mimic ADSCs group), IL-1 + exos from miR-486-5p mimic NC transfected ADSCs 806 (mimic NC exos group) and IL-1 $\beta+$ exos from miR-486-5p mimic transfected ADSCs 807 (miR-486-5p mimic exos group). (A) The apoptosis rate of each group was evaluated 808 using Annexin-V Staining. (B) The expression of CHOP of each group was evaluated 809 using immunofluorescent staining. (C) The expression of cleaved Caspase-3, GRP78, 810 type II collagen and MMP-13 were evaluated using Western Blot. GAPDH served as 811 internal control. (D) The concentration of IL-6 and TNF- $\alpha$ in culture media were 812 evaluated using ELISA. The statistical significance was assessed using one-way 813 ANOVAs with Tukey's multiple comparison tests. Data represent mean $\pm S D\left({ }^{*} p<0.05\right.$, $\left.{ }^{* *} p<0.01,{ }^{* * *} p<0.001\right)$ 
A
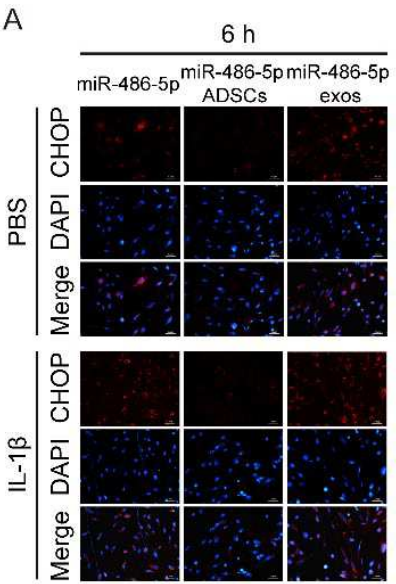
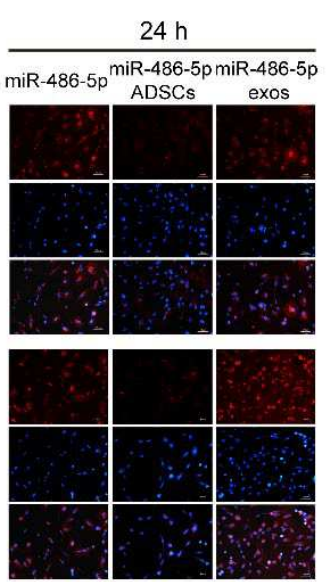
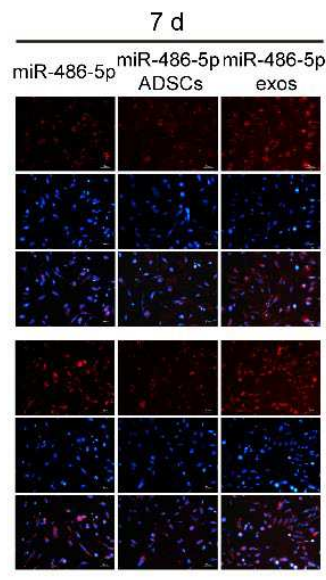
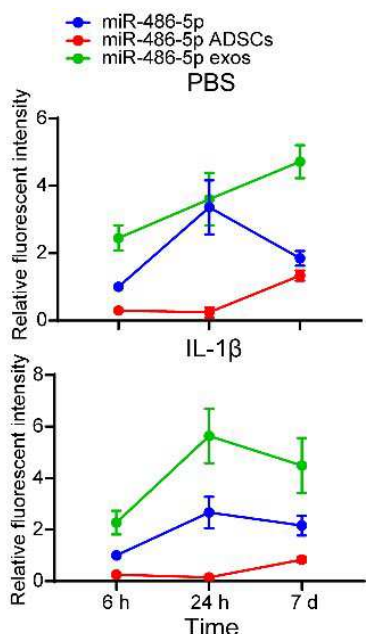

DMM

B

Normal

miR-486-5p miR-486-5p ADSCs miR-486-5p exos
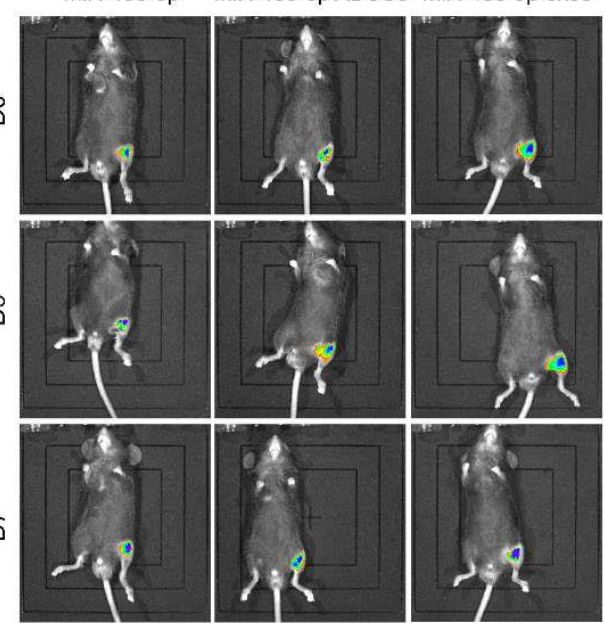

miR-486-5p miR-486-5p ADSCs miR-486-5p exos
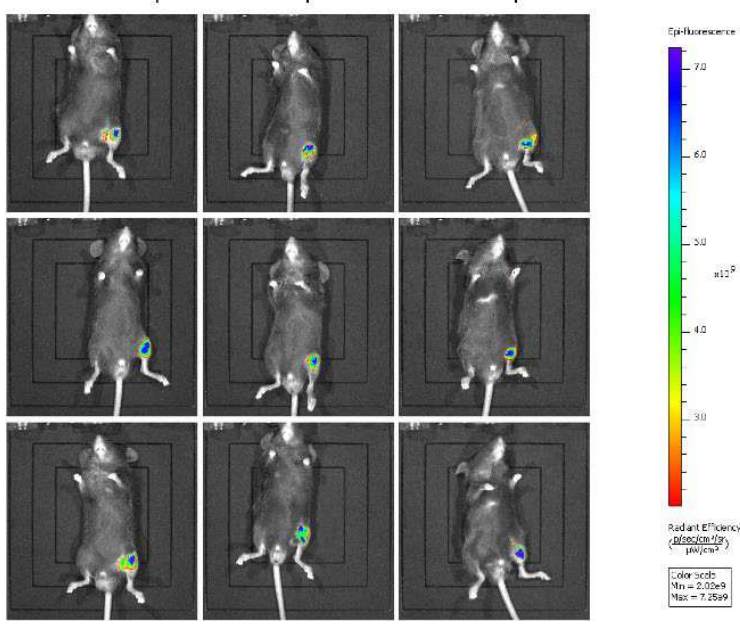

Fig. 7 Tracking of different miR-486-5p administrative methods in vitro and in

vivo. miR-486-5p was labeled with fluorescent dye Cy3 (miR-486-5p-Cy3) and miR486-5p-Cy3 transfected ADSCs (miR-486-Cy3 ADSCs), and exos from miR-486-5pCy3 transfected ADSCs (miR-486-Cy 3 exos) were obtained. (A) In vitro, noninflammatory and inflammatory environments $(10 \mathrm{ng} / \mathrm{mL} \mathrm{IL}-1 \beta)$ were created for chondrocytes before they were treated with miR-486-5p-Cy3, miR-486-5p-Cy3 ADSCs and miR-486-5p-Cy3 exos. After 6 h, 24 h and 7 days, the uptake rate of miR-486-5pCy3 was measured using fluorescent staining. (B) In vivo, non-inflammatory and inflammatory knee joint micro-environments (DMM) were created before injection with miR-486-5p-Сy3, miR-486-5p-Сy3 ADSCs and miR-486-5p-Сy3 exos. The fluorescent 
A

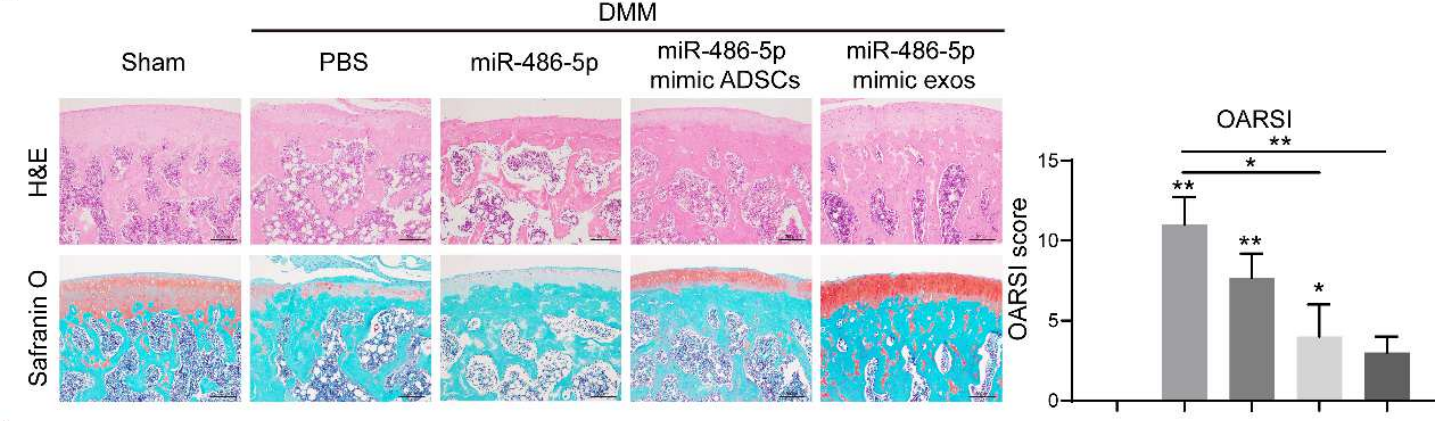

B

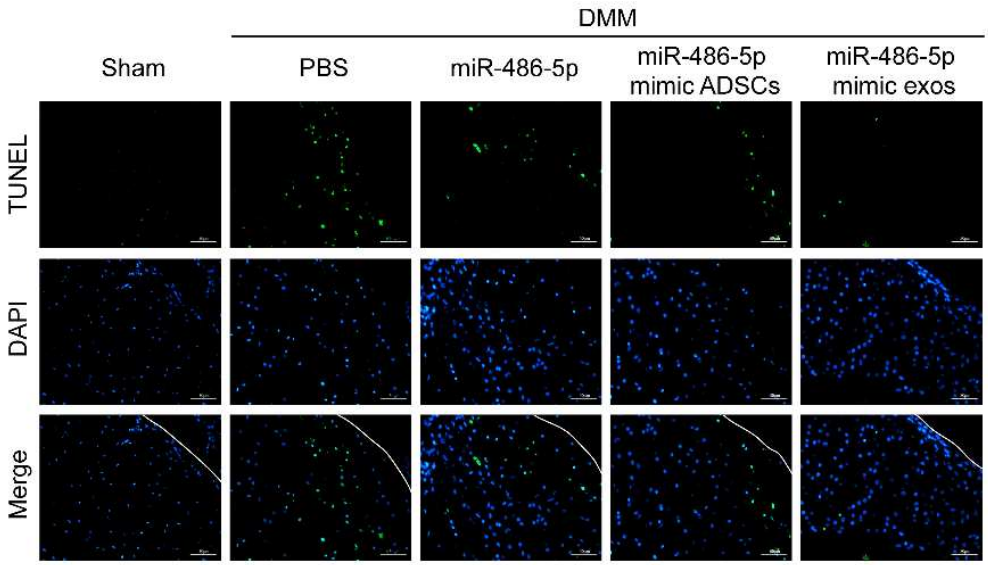

C
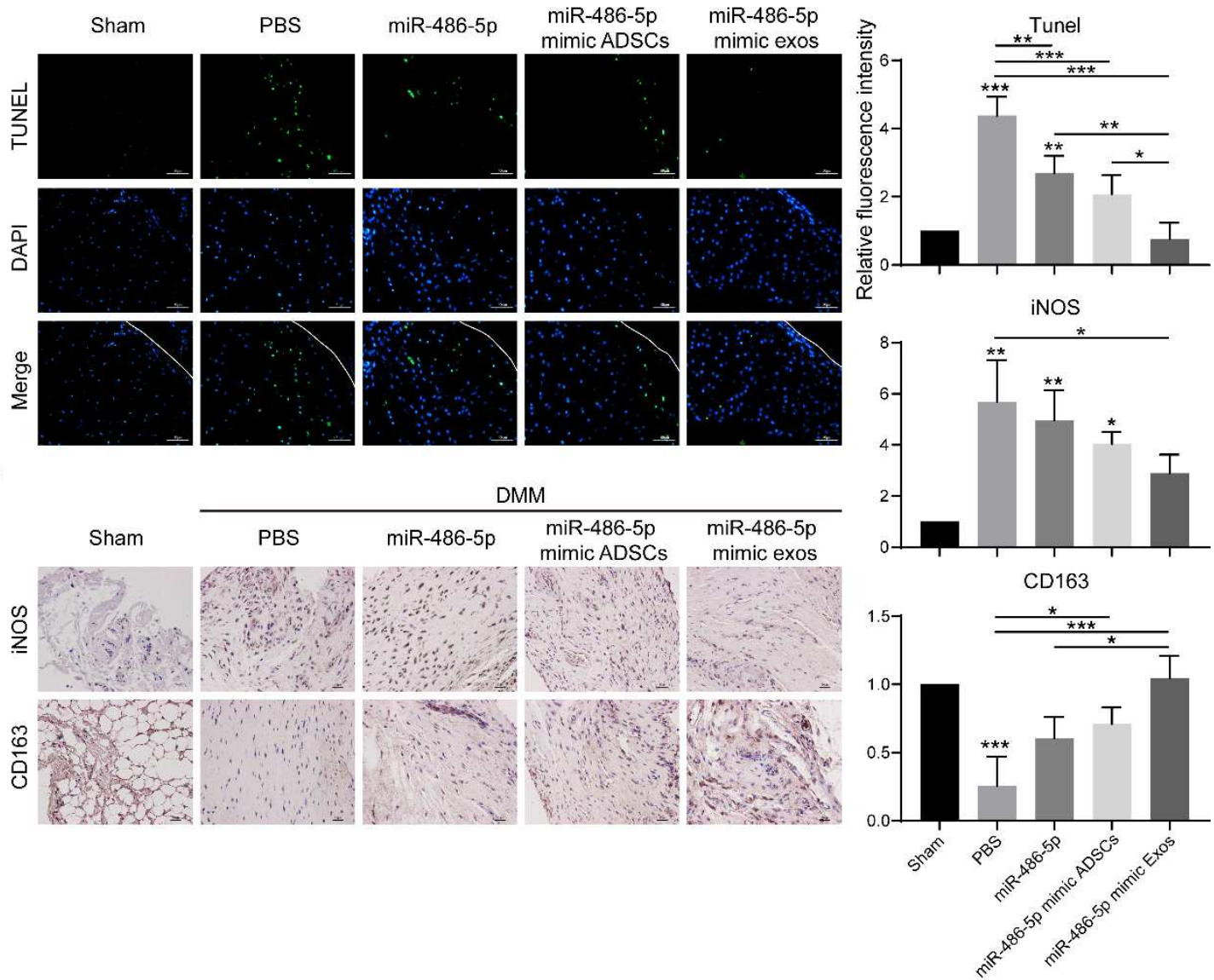

Fig. 8 In vivo effect of different miR-486-5p administrative methods in OA mice

model. OA model (DMM) was set up and miR-486-5p mimic (miR-486-5p group), miR486-5p transfected ADSCs (miR-486-5p mimic ADSCs group), and exos from miR486-5p transfected ADSCs (miR-486-5p mimic exos group) were injected into the knee joint of normal and OA mice. Joint and synovium samples were collected after 10 weeks. (A) Hematoxylin-eosin (H\&E) and safranin O/fast green were used to evaluate 
834 the matrix deposition and cartilage erosion of joint samples. (B) TUNEL assay were 835 used to evaluate the apoptosis condition of joint samples. (C) Immunohistochemical 836 staining of iNOS and CD163 were used to evaluate the polarization of macrophages 837 in synovium samples.

838 